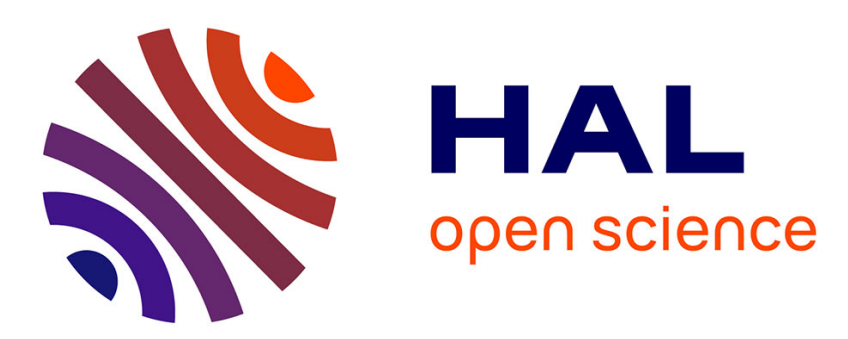

\title{
La réorganisation de la Crète après la bataille d'Actium. Le rôle de M. Nonius Balbus, patron de province
}

Sabine Lefebvre

\section{To cite this version:}

Sabine Lefebvre. La réorganisation de la Crète après la bataille d'Actium. Le rôle de M. Nonius Balbus, patron de province. KTÈMA Civilisations de l'Orient, de la Grèce et de Rome antiques, 2013, $\mathrm{n}^{\circ} 38$, p. 249-272. halshs-00967457

\section{HAL Id: halshs-00967457 https://shs.hal.science/halshs-00967457}

Submitted on 8 Jun 2021

HAL is a multi-disciplinary open access archive for the deposit and dissemination of scientific research documents, whether they are published or not. The documents may come from teaching and research institutions in France or abroad, or from public or private research centers.
L'archive ouverte pluridisciplinaire HAL, est destinée au dépôt et à la diffusion de documents scientifiques de niveau recherche, publiés ou non, émanant des établissements d'enseignement et de recherche français ou étrangers, des laboratoires publics ou privés. 


\title{
La réorganisation de la Crète après la bataille d'Actium. Le rôle de M. Nonius Balbus, patron de province
}

Sabine Lefebvre

\begin{abstract}
Following the conflict between Octavian and Marcus Antonius, and within the framework of the Augustan reform, the provincials had to show their reunification to the princeps, all the more if they lived in territories submitted to Marcus Antonius before the battle of Actium. To show their loyalty, the Cretans, with their koinon, chose a patronus prouinciae, M. Nonius Balbus, who was their governor. To replace this nomination in the political context, it is advisable to return to the fasti of the provinces of Crete and Cyrenaica between 31 and 27 B. C.
\end{abstract}

\section{Résumé}

À la suite du conflit opposant Octavien et Marc Antoine, et dans le cadre de la réforme augustéenne, les provinciaux ont dû manifester leur ralliement au princeps, d'autant plus s'ils relevaient des territoires soumis à Marc Antoine avant la bataille d'Actium. Afin de manifester leur fidélité, les Crétois, par le biais de leur koinon, ont choisi un patronus prouinciae, M. Nonius Balbus, qui fut leur gouverneur. Afin de replacer cette cooptation dans le contexte politique, il convient de revenir sur les fastes des provinces de Crète et de Cyrénaïque entre 31 et 27 av. J.-C.

\section{Citer ce document / Cite this document :}

Lefebvre Sabine. La réorganisation de la Crète après la bataille d'Actium. Le rôle de M. Nonius Balbus, patron de province. In: Ktèma : civilisations de l'Orient, de la Grèce et de Rome antiques, N³8, 2013. La question des pauvres et de la pauvreté dans le monde grec. pp. 249-272;

doi : https://doi.org/10.3406/ktema.2013.1412

https://www.persee.fr/doc/ktema_0221-5896_2013_num_38_1_1412

Fichier pdf généré le 07/05/2021 


\title{
La réorganisation de la Crète après la bataille d'Actium
}

\author{
Le rôle de M. Nonius Balbus, patron de province*
}

\begin{abstract}
RÉsumé-. À la suite du conflit opposant Octavien et Marc Antoine, et dans le cadre de la réforme augustéenne, les provinciaux ont dû manifester leur ralliement au princeps, d'autant plus s'ils relevaient des territoires soumis à Marc Antoine avant la bataille d'Actium. Afin de manifester leur fidélité, les Crétois, par le biais de leur koinon, ont choisi un patronus prouinciae, M. Nonius Balbus, qui fut leur gouverneur. Afin de replacer cette cooptation dans le contexte politique, il convient de revenir sur les fastes des provinces de Crète et de Cyrénaïque entre 31 et 27 av. J.-C.
\end{abstract}

Abstract-. Following the conflict between Octavian and Marcus Antonius, and within the framework of the Augustan reform, the provincials had to show their reunification to the princeps, all the more if they lived in territories submitted to Marcus Antonius before the battle of Actium. To show their loyalty, the Cretans, with their koinon, chose a patronus prouinciae, M. Nonius Balbus, who was their governor. To replace this nomination in the political context, it is advisable to return to the fasti of the provinces of Crete and Cyrenaica between 31 and 27 B. C.

La mise en place du régime augustéen, après la victoire d'Actium, a dû tenir compte de la diversité des situations tant à Rome que dans les provinces. Si les provinces occidentales avaient prêté serment à Octavien dès 32 av. J.-C. ${ }^{1}$, les provinces relevant de Marc Antoine, dans les quelques années qui ont suivi la mort de ce dernier, ont dû craindre des représailles, ou tout au moins un traitement plus rude de la part du princeps. Les témoignages sont rares qui évoquent ces craintes et les réponses ponctuelles apportées par le pouvoir central.

Néanmoins un dossier, mieux documenté, permet de voir comment a pu s'établir un dialogue entre les populations provinciales et Octavien-Auguste: il s'agit du cas de la Crète, confiée à M. Nonius Balbus, gouverneur nommé par le Prince dans ce contexte particulier. Nommé proconsul de Crète en 29/28 av. J.-C. ${ }^{2}$, ce dernier est originaire de Campanie ${ }^{3}$. Le choix d'Octavien fut sans doute justifié par la fidélité de cet homme, dont la carrière a été suivie par le jeune imperator. Mais si ce dernier choisit d'envoyer un fidèle en Crète, c'est parce que la province avait été très marquée par la présence de Marc Antoine; il fallait donc rétablir l'ordre, reprendre en main la population,

${ }^{*}$ ) Je remercie Bernard Legras et Fabrice Poli, qui ont relu ce texte.

(1) RGDA, 25.

(2) Cf. notre analyse ci-dessous.

(3) Pour un dernier état de la bibliographie et de la documentation le concernant, cf. Lefebvre S., Patronus prouinciae, à paraître. 
et en particulier s'assurer de sa fidélité et surtout de celle des élites locales. Ces dernières sont sans nul doute tout à fait au courant de la carrière de leur proconsul et des intentions d'Octavien. Afin de passer en douceur ce cap difficile, il leur a paru utile de choisir un protecteur qui saurait intercéder en leur faveur auprès du Prince. Choisir un notable local était impossible: trop peu avaient accédé à la citoyenneté romaine, même si on doit prendre en compte la toute jeune colonie de Cnossos; de plus, la plupart étaient sans doute marqués par leur attachement antérieur à Marc Antoine et ne disposaient donc pas des relais nécessaires à Rome pour entrer en contact avec Octavien. Choisir un ancien gouverneur posait aussi problème: depuis plus de dix ans, la Crète ne relevait plus d'un responsable officiel romain. Il aurait alors fallu avoir recours à des gouverneurs plus anciens, mais les Crétois n'en avaient peut-être pas gardé de bons souvenirs, la plupart étaient sans doute morts lors des guerres civiles - ou de leur belle mort. L'unique solution qui s'offrait à eux était de recourir aux bons offices du gouverneur en poste, M. Nonius Balbus. Ce dernier connaissait les responsabilités du patron; il assumait en effet le patronat d'Herculanum ${ }^{4}$, où il avait accompli de nombreux actes d'évergétisme et où il devait, par la suite, être enterré.

Malheureusement, les textes ${ }^{5}$ dont nous disposons ne mentionnent pas les services rendus à la Crète par son patron. Cependant, les cinq hommages rendus par les Crétois ${ }^{6}$ témoignent de la réussite de $\mathrm{M}$. Nonius Balbus, et du soulagement de sa province cliente. Il est significatif que, par la suite, les problèmes étant réglés, les provinciaux n'aient plus ressenti le besoin d'avoir un puissant protecteur. Aussi, l'hypothèse de M.A. Baldwin ${ }^{7}$, expliquant deux au moins des hommages ${ }^{8}$ par

(4) Maiuri A., Atti della reale Accademia d'Italia, Rendiconti della classe di Scienze morali e storiche, ser. 7, III, 1943, p. 253-278 (AE, 1947, 53) = Schumacher L., Chiron, 6, 1976, p. 165-184 (AE, 1976, 144) Herculanum.

(5) Les inscriptions et les monnaies utilisées seront mentionnées selon les abréviations suivantes, qui renvoient également aux ouvrages de prosopographie :

Abréviations des corpora épigraphiques

AE = L'Année épigraphique, Paris, 1888-

$C I L=$ Corpus Inscriptionum Latinarum, Berlin, 1863-.

IGRR, I = Cagnat R., Toutain J. et Jouguet P., Inscriptiones Graecae ad res Romanas pertinentes, I, Paris, 1911.

I. Cret. = GuARDUCCI M., Inscriptiones Creticae, Rome, 1935/50.

ILS = Dessau H., Incriptiones latinae selectae, Berlin, 1892/1916.

CIG, II = Bоескн A., Corpus Inscriptionum Graecarum, II, Berlin, 1843.

MAMA, VIII = CALDER W. M. et CORMACK J. M. R., Monuments from Lycaonia, the Pisido-Phrygian Borderland, Aphrodisias,

Monumenta Asiae Minoris Antiqua, Manchester, 1962.

RGDA = ScheID J. (trad..), Res gestae divi Augusti, CUF, Paris, 2007.

Abréviations des corpora numismatiques

BMC Cret. = Wrotн W., Catalogue of the Greek Coins of Crete and the Aegean Islands in the British Museum, Londres, 1886. BMC Cyr. = Robinson E. S. G., Catalogue of the Greek Coins of Cyrenaica in the British Museum, Londres, 1927.

BMC Emp., I = Mattingly H., Coins of the Roman Empire in the British Museum, I, Augustus to Vitellius, Londres, 1923. BMC Rep., II = Grueber H.A., Coins of the Roman Republic in the British Museum, Oxford, 1910.

CBN, I = Giard J.-B., Bibliothèque nationale: Catalogue des monnaies de l'Empire romain, I, Paris, 1976.

RIC, $\mathrm{I}^{2}=$ Sutherland C. H. V., The Roman Imperial Coinage, I, From 31 B.C. to 69 A.D., Londres, 1984.

$R P C, \mathrm{I}=$ Burnett A., Amandry M., Pau Ripollès P., Roman Provincial Coinage, I. From the Death of Caesar to the Death of Vitellius (44 BC-AD 69), Londres-Paris, 1992.

RRC = CrawFord M., Roman Republican Coinage, Cambridge, 1974.

Abréviations des ouvrages prosopographiques

$M R R=$ Broughton T.R.S., The Magistrates of the Roman Republic, II, New York, 1952.

MRR, Sup. = Broughton T.R.S., Supplement to the Magistrates of the Roman Republic, New York, 1960.

$M R R$, III = Broughton T.R.S., The Magistrates of the Roman Republic, III, Atlanta, 1986.

$P I R^{2}=$ Prosopographia Imperii Romani, saec. I, II, III, Berlin, 1933.

(6) Cf. annexe 1.

(7) Baldwin M. A., Fasti, 1983, p. 49;83-88, nº 2.

(8) Cf. annexe 1, doc. d; e. 
le règlement de tensions liées à la mise en place du territoire de plusieurs cités, ne paraît-elle pas devoir être prise en compte; en effet, ce type de situation est fréquent, et n'a pas ailleurs entraîné les mêmes réactions de la part des provinciaux.

Il convient donc de revenir rapidement sur la situation crétoise pour bien comprendre les enjeux de cette nomination. J'évoquerai ensuite la reprise en main augustéenne et les réactions provinciales, pour enfin évoquer le choix de M. Nonius Balbus par le koinon crétois.

\section{VERS UNE CRÈTE PRO-ANTONIENNE}

La Crète est un territoire entré tardivement dans l'Empire romain, même si les premières rencontres diplomatiques remontent à $195 \mathrm{av}$. J.-C. ${ }^{9}$, et se poursuivent par la suite. Dès cette date, les choix politiques et diplomatiques des cités crétoises témoignent d'une organisation, par exemple lors de l'aide apportée aux pirates en $71 / 70$ av. J.-C. ${ }^{10}$. La guerre est engagée, menée par le proconsul Cretae et Achaiae ${ }^{11}$, Q. Caecilius Metellus Creticus ${ }^{12}$, dont les succès militaires lui valent d'être salué imperator ${ }^{13}$ en 67 , et de porter le cognomen Creticus $^{14}$.

Désormais devenue province ${ }^{15}$, la Crète est parfois associée à la Cyrénaïque ${ }^{16}$, léguée à Rome en 96 av. J.-C. ${ }^{17}$, mais constituée comme province en 74 av. J.-C. seulement ${ }^{18}$. Elles seraient alors gouvernées conjointement par un sénateur; mais cette association n'est pas attestée de façon permanente entre 66 av. J.-C. et l'arrivée de Marc Antoine ${ }^{19}$. Ainsi, à partir de

(9) Tite Live, XXXIV, 35; cf. van Effenterre H., Crète, 1948, p. 257-260 sur la guerre contre Nabis, tyran de Sparte et les répercutions sur la Crète; Willets R.F., Society, 1955, p. 237; SAnders I. F., Crete, 1982, p. 3; Luzzatto G. I., Roma, 1985, p. 152-153.

(10) Strabon, X, 4, 9; Justin, XXXIX, 5, 3.

(11) Tite Live, Per., LXXXXIIX, 7; Cicéron, Ad Brut., I, 8 (DccCcviII) en mai/juin 43 av. J.-C.; Plutarque, Pompée, 29, 2; Valère Maxime, VII, 6, ext 1; Florus, I, 42, 4; Appien, Sic., 6, 2 ; Phlegon de Tralles, Frg. 12, FGH, 2, B, p. $1163-1164$; Dion Cassius, XXXVI, 1; Eutrope, VI, 11, 1-2; Orose, VI, 4, 2.

(12) Il est consul en 69 av. J.-C.; PARIBEnI R., dans DE, II, 2, 1910, s. u. Creta, col. 1263-1264; MRR, II, p. 139; 145; 154; Perl G., Klio, 52, 1970, p. 330, n 3; ID., Klio, 53, 1971, p. 370-371; Sherwin-White A. N., CAH², IX, 1994, p. 249.

(13) CIL, I², 2, 746 Merbakae; IGRR, I, 955 = I. Cret, 2, p. 252, nº 14 Polyrhénion; Cicéron, Flacc., 63; Tite Live, Per., XCIX, 1; 4; Dion Cassius, XXXVI, 19, 3. Cf. Svoronos J., Numismatique, 1890, p. 276; Sherwin-White A. N., CAH², IX, 1994 , p. 250.

(14) Florus, I, 42 (III, 7).

(15) Cicéron, Flacc., 100.

(16) Paribeni R., dans DE, II, 2, 1910, s. u. Creta, col. 1264; Romanelli P., CAH, XI, 1936, p. 649; Romanelli P., Cirenaica, 1943, p. 50-51; Larsen J. A. O., Classical Philology, 47, 1952, p. 8-9; Perl G., Klio, 52, 1970, p. 319-354; ID., Klio, 53, 1971, p. 369-379; Bertrand J.-M., dans Rome et la conquête du monde méditerranéen, 2, 1978, p. 814; SANDERs I. F., Crete, 1982, p. 4-5; LARonde A., Cyrène, 1987, p. 478-479. Elles seraient séparées entre 52 et 49, puis entre 44 et 43 av. J.-C.. Pour A. Chapman (Num. Chron., 8, 1968, p. 13), elles sont associées de 67 à 44 av. J.-C.

(17) Tite Live, Per., LXX, 5; Justin, XXXIX, 5. Cf. Oоsт S. I., Classical Philology, 58, 1963, p. 11-25; Luzzatto G. I., Roma, 1985, p. 146-148; Buttrey T. V., dans The Coinage of the Roman World in the Late Republic, 1987, p. 165-166; Laronde A., Cyrène, 1987, p. 455 et s.; Laronde A., ANRW, II, 10, 1, 1988, p. 1007-1011; Thompson D. J., CAH², IX, 1994, p. 316; Reynolds J. et Llyod J. A., $C A H^{2}$, X, 1996, p. 619.

(18) Salluste, Hist. Reliquae, II, 43; Appien, BC, I, 111. Cf. Romanelli P., Cirenaica, 1943, p. 43; 46, note 2; Perl G., Klio, 52, 1970, p. 319-325; ID., Klio, 53, 1971, p. 369-370; Luzzatto G. I., Roma, 1985, p. 146-149; Thompson D. J., CAH², IX, 1994, p. 319; ReYNolds J. et Llyod J. A., $C A H^{2}$, X, 1996, p. 621. Le premier gouverneur connu est P. Cornelius Lentulus Marcellinus; il serait intervenu lors de la guerre contre les pirates en tant que légat de Pompée. Sur ce premier gouverneur de Cyrénaïque, cf. Perl G., Klio, 52, 1970, p. 327-329, n 1 et 2; Laronde A., Cyrène, 1987, p. 478.

(19) Même Cn. Tremellius Scrofa aurait été gouverneur de l'une OU de l'autre des deux provinces. Cf. Cicéron, Att., VI, 1, 13 (cCXlv) en février 50 av. J.-C.: Iam Scrofa uellem haberet ubi posset: est enim lautum negotium (mais le texte ne mentionne aucun gouvernement). Cf. Perl G., Klio, 52, 1970, p. 333, nº 7; SANders I. F., Crete, 1982, p. 5 ; 176. 
juillet 44 av. J.-C. ${ }^{20}$, la Cyrénaïque est confiée à C. Cassius Longinus ${ }^{21}$, alors que la Crète est attribuée à M. Iunius Brutus ${ }^{22}$, qui en confie l'administration à P. Aemilius Lepidus en 43 av. J.-C. ${ }^{23}$. Aussi certains pensent-ils que cette association «ne semble pas antérieure à Marc Antoine ${ }^{24}$; en effet, c'est ce dernier qui les réunit effectivement entre $40^{25}$ et 34 av. J.-C., date à laquelle la Cyrénaïque est donnée à Cléopâtre Séléné ${ }^{26}$. L'administration de la Crète change alors de mains.

S'étant vu confier par ses collègues triumvirs la partie orientale de l'Empire qu'il administre totalement dès 42 av. J.-C. ${ }^{27}$, Marc Antoine a commencé à réorganiser ces régions assez tôt, y envoyant par exemple L. Lollius ${ }^{28}$, bien attesté en Cyrénaïque vers 39 av. J.-C. ${ }^{29}$ ainsi qu'en Crète ${ }^{30}$. Mais c'est à partir de 37 av. J.-C. que les mesures principales sont prises ${ }^{31}$, avec un premier partage en 37/36 av. J.-C. ${ }^{32}$ : Cléopâtre et ses enfants se voient confier une partie de la Crète, la Cyrénaïque et Chypre $^{33}$, en particulier la cité d'Itanos, avec qui l'Égypte entretient des relations militaires depuis déjà plusieurs années ${ }^{34}$. Le partage de l'automne 34 av. J.-C. confirme ces arrangements pour la

(20) Appien, BC, III, 8; Plutarque, Brutus, 19, 5. Cf. Sternkopf W., Hermes, 47, 1912, p. 341; Harrison G., Cretan Studies, 1, 1988, p. 143.

(21) Sternkopf W., Hermes, 47, 1912, p. 342-349; 381-385; Perl G., Klio, 52, 1970, p. 333-334, nº 9.

(22) Cicéron, Phil., II, 38, 97. Cf. Imhoof-Blumer F., Monnaies, 1883, p. 212; Sternkopf W., Hermes, 47, 1912, p. 342-349; 381-385; Raven E. J.P., Num. Chron., 18, 1938, p. 146; Perl G., Klio, 52, 1970, p. 334, n 10; 342; ID., Klio, 53, 1971, p. 373.

(23) Appien, BC, V, 2; Cicéron, Phil., XI, 12, 27. Sa présence est également attestée par des monnaies; sur le débat le concernant, $\mathrm{cf}$. annexe 2. Les monnaies du pseudo P. Licinius.

Sur la présence de P. Lepidus, cf. Raven E. J.P., Num. Chron., 18, 1938, p. 146-147; Romanelli P., Cirenaica, 1943, p. 62; Charlesworth M.P., CAH, X, 1963, p. 9, note 3; Bowersock G., Augustus, 1965, p. 18; Perl G., Klio, 52, 1970, p. 336-337, n $^{\circ} 13$; pour P. Lepidus, p. 337-338, n $14 ; 342$, sous le nom de Licinius et de Lepidus; SANDERs I. F., Crete, 1982, p. 5; 178; Luzzatto G. I., Roma, 1985, p. 149; 154; Reynolds J. et Llyod J.A., $C A H^{2}$, X, 1996, p. 630; CABANES P., dans Rome et l'intégration de l'Empire 44 av. J.-C./260 ap. J.-C., 2, 1998, p. 304 ; ZucCA R., dans La Cirenaica in età antica, 1998, p. 624. Pour Lepid(us) (Grant M., Imperium, 1946, p. 35), ou Lepid(ius), cf. Price M., INJ, 6/7, 1982/83, p. 119-120; Buttrey T.V., dans The Coinage of the Roman World in the Late Republic, 1987, p. 168, note 7.

(24) Perl G., Klio, 52, 1970, p. 342: «und von einer Vereinigung mit Cyrenae nicht die Rede»; Harrison G., Cretan Studies, 1, 1988, p. 141; 142; Reynolds J. et Llyod J.A., CAH², X, 1996, p. 621: «it is not certain that Cyrenaica was governed with Crete at this stage»; Rémy B., Karthago, 24, 1999, p. 165 suivant PerL G., Klio, 52, 1970, p. 326-327, et Laronde A., Cyrène, 1987, p. 479; Laronde A., ANRW, II, 10, 1, 1988, p. 1013.

(25) À cette date est connu par des monnaies le gouverneur P. Lepidius, anciennement connu sous le nom de P. Licinius (cf. ci-dessus, note 22). Vers 40 av. J.-C., il a clairement en charge la Cyrénaïque et la Crète, les types monétaires portant soit

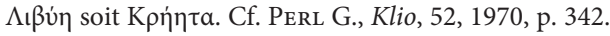

(26) Romanelli P., Cirenaica, 1943, p. 63.

(27) Reynolds J. et Llyod J. A., CAH'2, X, 1996, p. 630.

(28) PIR², L, 319; Perl G., Klio, 52, 1970, p. 338-339, nº 15; 342; SAnders I. F., Crete, 1982, p. 5; 176; Chapman A., Num. Chron., 8, 1968, p. 15-16. Cf. Bowersock G., Augustus, 1965, p. 23; 27-28.

(29) Pour la date de sa présence, certains proposent entre 36 et 34 av. J.-C. (MRR, II, p. 582; MRR, Sup., p. 36; MRR, III, p. 127; RPC, I, p. 219), alors que d'autres penchent pour 39 av. J.-C. ou juste après (BABELON E., Monnaies, 1885, II, p. 149: "antérieures au règne d'Auguste»; BMC Cyr., p. ccxvi; Chapman A., Num. Chron., 8, 1968, p. 16; Sanders I. F., Crete, 1982, p. 176), voire pour la période pré-antonine (Buttrey T.V., dans Studies in Numismatic Method presented to Philip Grierson, 1983, p. 30).

(30) Grant M., Imperium, 1946, p. 56-58; Chapman A., Num. Chron., 8, 1968, p. 15-16: pour elle, la légende en latin signifierait que la frappe est à relier à la Crète, où les monnaies auraient été frappées. Cf. Chapman A., Num. Chron., 8, 1968, p. 15, pl. V, n 4-8; Buttrey T. V., dans Studies in Numismatic Method presented to Philip Grierson, 1983, p. 24-25; ID., dans The Coinage of the Roman World in the Late Republic, 1987, p. 168-169; RPC, I, p. 218-219, n 908-913.

(31) SArtre M., Orient, 1991, p. 13.

(32) Freyburger M.-L. et Roddaz J.-M., Dion Cassius, 48-49, 1994, p. CXXIV-CXXVI.

(33) Plutarque, Antoine, 36, 3; Dion Cassius, XLIX, 32, 5; Porphyre de Tyr dans Eusèbe, FHG, 2, B, p. 724, §9. Cf. Grant M., Imperium, 1946, p. 55; Spyridakis S, Itanos, 1970, p. 87; Pelling C., CAH², X, 1996, p. 29; Levick B. M., $C A H^{2}, \mathrm{X}, 1996$, p. 647.

(34) Une garnison égyptienne se trouvait à Itanos, mais à la conquête romaine les troupes ont quitté l'île; cf. SPYRIDAKIS S., Itanos, 1970. 
Crète et la Cyrénaïque ${ }^{35}$, si ce n'est que la Cyrénaïque relève désormais de la tutelle toute théorique de la petite Cléopâtre Séléné ${ }^{36}$. Une monnaie découverte à Cyrène, datée de 31 av. J.-C. et montrant Antoine et Cléopâtre, pourrait laisser penser que la titulaire du royaume serait en fait Cléopâtre elle-même ${ }^{37}$. Mais Marc Antoine continue à en assurer le contrôle administratif et militaire en y plaçant quatre légions sous les ordres de L. Pinarius Scarpus ${ }^{38}$. Quant à la Crète, bien qu'elle soit théoriquement rendue à la liberté par Marc Antoine ${ }^{39}$, elle est en fait toujours sous le contrôle de Rome comme en témoigne le passage d'officiels romains, comme Crassus en 37/36 av. J.-C., dont la présence dans cette région n'est connue que par les monnaies ${ }^{40}$.

Dans le même temps, Octavien aurait accordé à la cité de Capoue des terres situées en Crète, sur le territoire de $\operatorname{Cnossos}^{41}$, en partie détruite en 67 av. J.-C. et qui en compensation est promue colonie $^{42}$. Selon certains ${ }^{43}$, P. Canidius Crassus aurait été le deductor de la nouvelle colonie.

Mais la bataille d'Actium remet en cause cette organisation, et alors que les principaux alliés de Marc Antoine se sont rangés à ses côtés lors du conflit qui l'oppose à Octavien ${ }^{44}$, la tâche du vainqueur est de reprendre en main les provinces orientales, en particulier la Crète.

(35) Plutarque, Antoine, 54, 6; Dion Cassius, L, 25, 4. Cf. DoвıÁs J., dans Mél. Bidez, I, 1934, p. 287-314: la Cyrénaïque n'aurait été donnée à Cléopâtre qu'en 34 av. J.-C.; Pelling C., CAH², X, 1996, p. 40.

(36) Dion Cassius, XlIX, 41, 2. Cf. Romanelli P., Cirenaica, 1943, p. 57; Luzzatto G.I., Roma, 1985, p. 149; Chamoux F., Marc Antoine, 1986, p. 364; Reynolds J. et Llyod J. A., CAH², X, 1996, p. 630.

(37) Müller L., Supplément, 1874, p. 30, n 428a = Svoronos J.N., Ptolémées, 1904, p. 317, nº 1899, pl. LXIII, $26=$ Regling K., ZfN, 25, 1906, p. 397, nº 1899-1900 = Grant M., Imperium, 1946, p. 64-65, pl. 1, 20 mais il l'attribue à Patras; T. V. Buttrey (ANSMN, 1954, p. 95-109) la place encore à Patras, mais il est revenu sur cette opinion (ButTREy T. V., dans Studies in Numismatic Method presented to Philip Grierson, 1983, p. 32) ; RPC, I, p. 221; p. 222, n 924.

(38) Reynolds J. et Llyod J. A., CAH², X, 1996, p. 631; cf. ci-dessous, p. 256.

(39) Un décret de Marc Antoine, promulgué en 44 av. J.-C., (et sans doute préparé par César) et appliqué en 43 av. J.-C., aurait exempté certaines cités crétoises de la taxe et mis fin au système provincial en ce qui concerne cette province. Il s'agit d'un faux, comme en témoigne Cicéron, Phil., II, 38, 97: Nuper fixa tabula est, qua ciuitates locupletissimae Cretensium uectigalibus liberantur statuiturque ne post M. Brutum pro consule sit Creta prouincia ... An Caesaris decreto Creta post M. Bruti decessum potuit liberari, cum Creta nihil ad Brutum Caesare uiuo pertineret?; cf. aussi Dion Cassius, XLIX, 32, 4. La rumeur a sans doute néanmoins fait beaucoup pour la popularité de Marc Antoine dans l'île, qu'il contrôle totalement après la bataille de Philippes en 42 av. J.-C.. Cf. PerL G., Klio, 52, 1970, p. 342, nº 18.

(40) BMC Cyr., p. ccvi-ccvii et pl. XLII 10-XLIV 3; p. 117; 127 pour les monnaies en grec. Il existe aussi une série en latin, qui aurait été plus spécifiquement frappée en Crète (p. ccxxi-ccxxii); CHAPmAN A., Num. Chron., 8, 1968, p. 15, pl. I, $\mathrm{n}^{\circ} 1-4$; Perl G., Klio, 52, 1970, p. 339-340, nº 16; Sanders I. F., Crete, 1982, p. 5; 176; 14; RPC, I, p. 220, n 914, pl. 51, $\mathrm{n}^{\circ}$ 914. Les monnaies portant l'inscription PTOLEMAI[S] en l'honneur de la cité de Cyrène et un crocodile, symbole de l'Égypte avec une seconde inscription KRAS ou KRA ont sans soute été frappées en Cyrénaïque (RPC, I, p. 220-221, $\mathrm{n}^{\circ}$ 916-918, pl. 51, n 916-918). Cf. Buttrey T. V., dans Studies in Numismatic Method presented to Philip Grierson, 1983, p. 25. Certaines monnaies ont été surfrappées par Kydas.

(41) Ducrey P., BCH, 93, 1969, p. 846-852, n³ (AE, 1969/1970, 635) Archanes: [I]mp(eratore) Domitiano / Caesar(e) Aug(usto), Germ(anico), X co(n)(s(ule), / [i]nter col(oniam) Flau(iam) Aug(ustam) Feli(cem) / Cap(uam) et Plotium Plebeium, / [ex] senten(tia) Titi imp(eratoris) Aug(usti) item / [sec] und(um) decretum col(oniae) Cap(uae), / [ex c] onuentione u[tri]usq(ue) / [parti]s [t]ermini positi sunt[t], agente P(ublio) Mess[i]o Campano, / proc(uratore) [C]a[es]aris; Appien, BC, IV 1,3 (il présente les raisons pour lesquelles Capoue est lésée durant les premiers mois du triumvirat); Velleius Paterculus,

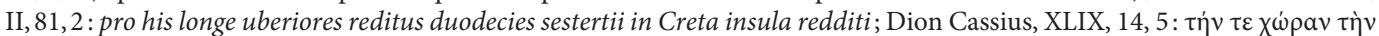
Kvwríav. Cf. Paribeni R., dans DE, II, 2, 1910, s. u. Creta, col. 1265; Perl G., Klio, 52, 1970, p. 343 ; Rigsby K. J., TAPA, 106, 1976, en particulier p. 329; SAnders I. F., Crete, 1982, p. 14; Luzzatto G. I., Roma, 1985, p. 156. Il ne s'agit bien entendu pas de donner à Capoue l'ensemble du territoire de Cnossos, contrairement à ce que pensait ImHoof-Blumer F., Monnaies, 1883, p. 211; Svoronos J., Numismatique, 1890, p. 64.

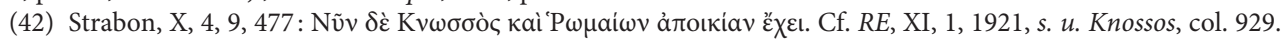

(43) Perl G., Klio, 52, 1970, p. 339-340, nº 16.

(44) Plutarque, Antoine, 61. Cf. Sartre M., Orient, 1991, p. 13. 
Après la bataille d'Actium, Octavien bénéficie de l'image positive laissée par son père adoptif et une politique fondée sur la clementia est attendue par les différents souverains des royaumes vassaux; mais les cités provinciales ne sont pas assurées de bénéficier d'une telle attitude. Aussi vont-elles, sans nul doute, être très attentives à la réorganisation générale de la partie orientale de l'Empire qui occupe Octavien au cours de l'année 30 av. J.-C. ${ }^{45}$, alors qu'il séjourne en Asie mineure, en particulier à Samos au cours de l'hiver 31/30 av. J.-C. Il s'agit de faire disparaître toute trace de l'activité du triumvir Marc Antoine ${ }^{46}$, en particulier dans les provinces romaines que ce dernier avait attribuées à la famille de Cléopâtre, et de remettre en ordre un «monde grec (qui) se trouvait dans une des situations les plus critiques de son histoire ${ }^{47}$ ».

Pendant trois ans, les divers gouvernements provinciaux sont confiés à des hommes sûrs, énergiques, capables de faire passer la fidélité des provinciaux de Marc Antoine à Octavien et de faire revenir la province de Crète dans le giron romain ${ }^{48}$. Ce moment est mal connu ${ }^{49}$ : les sources ne sont guère bavardes sur cette période courte mais néanmoins décisive. Ce n'est qu'en 27 av. J.-C. qu'Auguste revoit l'administration provinciale, faisant attribuer par le Sénat les provinces soit à sa propre administration, soit à celle du Sénat et du peuple romain ${ }^{50}$, dont relèvent la Crète et la Cyrénaïque ${ }^{51}$.

La Crète apparaît donc comme étant pro-antonienne jusqu'en 31 av. J.-C. au moins, sans que l'on sache quelle a été l'attitude de l'île - et de son koinon - pendant les préparatifs de la bataille d'Actium et les mois qui ont suivi la bataille; on peut supposer, en suivant A. Chapman ${ }^{52}$, que la situation est en effet restée incertaine jusqu'à la mort de Marc Antoine en 30. En effet, comme le rappelle $\mathrm{G}$. Bowersook ${ }^{53}$, «the shifts in allegiance among various Roman patrons had created an almost insoluble problem for many Greeks». La courte période ayant précédé le partage des provinces en 27 av. J.-C. ${ }^{54}$ a été l'occasion d'une reprise en main de l'île ${ }^{55}$; bien entendu, les distributions territoriales de Marc Antoine sont annulées ${ }^{56}$. L'attitude d'Octavien après sa victoire montre que tous les Crétois n'avaient pas dû se détourner de lui; en effet, il se montre généreux

(45) SArtre M., Orient, 1991, p. 14.

(46) Le système de relations établies avec les princes vassaux ne subit que des ajustements mineurs; cf. LEviCK B. M., $C A H^{2}$, X, 1996, p. $647 ; 649$.

(47) Roddaz J.-M., Agrippa, 1984, p. 427. Cf. Magie D., RRAM, 1950, p. 440-441; Bowersock G., Augustus, 1965 , p. 85.

(48) Levick B. M., $C A H^{2}$, X, 1996, p. 647.

(49) G. Perl (Klio, 52, 1970, p. 342) dit ainsi que «Das Schicksal Kretas zwischen 44 und 27 war wechselvoll und ist im einzelnen nicht ganz klar»! ; cf. récemment PAUt Asso A., ASAA, 72/73, 1994/95, p. 76: «il periodo che precede la definitiva sistemazione in provincia senatoria di rango pretorio, presenta numerosi punti oscuri ed ancora controversi ", que l'auteur choisit de ne pas aborder, débutant son étude en 27 av. J.-C.

(50) Harrison G., Cretan Studies, 1, 1988, p. 143; SArtre M., Orient, 1991, p. 14, note 4.

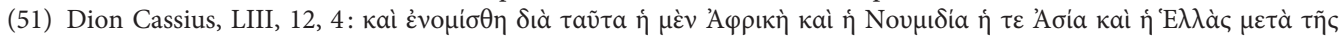

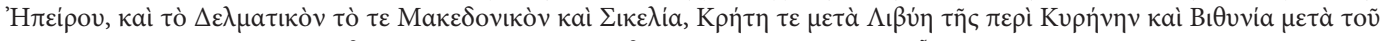

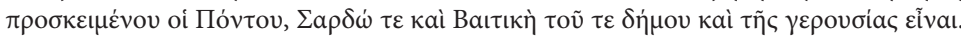

(52) Chapman A., Num. Chron., 8, 1968, p. 23: «a little after Actium, when the situation in the island would be one of uncertainty and before Octavian could do any organization ».

(53) Bowersock G., Augustus, 1965, p. 2.

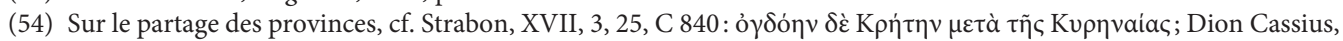
LIII, 12, 4. La Crète et la Cyrénaïque constituent à nouveau une seule et même province.

(55) Selon A. Chapman (Num. Chron., 8, 1968, p. 23-24), on peut dater de cette période deux séries de monnaies de bronze mentionnant des magistrats crétois (des crétarques?), Sauromatas et Aristion.

(56) RGDA, 31, 3: Prouincias omnis quae trans Hadrianum mare uergun[t a]d orientem Cyre/nasque iam ex parte magna regibus ea possidentibus ... reciperaui. Cf. TARN W. W. et CharlesworTH M. P., CAH, X, 1963, p. 114. 
envers trois cités ${ }^{57}$, Lampè, Kydonia, qui deviennent des cités libres, et Cnossos, villes qui lui avaient apporté leur aide, sans doute lors du changement de tutelle de l'île après la défaite de Marc Antoine. Mais certaines cités avaient dû rester favorables au vaincu, et, en ont donc subi les conséquences $^{58}$. Pour appliquer la rigueur nécessaire envers ces dernières, le Prince a sans doute veillé à ce que soient placés à la tête de la province des sénateurs fidèles et capables ${ }^{59}$, tout au moins dans les premières années. Mais les fastes de la province, qui se sont construits peu à peu ${ }^{60}$, ne sont pas clairs pour cette période; nous pouvons néanmoins tenter de les restituer pour la période charnière de 31 à 27 av. J.-C. dans le tableau ci-dessous :

Les fastes de la Crète et de la Cyrénaïque entre 31 et 27 av. J.-C.

\begin{tabular}{|c|c|c|}
\hline Date & Cyrénaique & Crète \\
\hline 31 av. J.-C. & L. Pinarius Scarpus en Cyrénaïque & \\
\hline 30 av. J.-C. & L. Pinarius Scarpus en Cyrénaïque & C. Rubellius Blandus ${ }^{59}$ en Crète? \\
\hline 29 av. J.-C. & $\begin{array}{l}\text { L. Pinarius Scarpus en Cyrénaïque? } \\
\text { L. Fabricius (= L. Fabricius Patellius?) }\end{array}$ & M. Nonius Balbus en Crète \\
\hline 28 av. J.-C. & L. Pinarius Scarpus en Cyrénaïque? L. Fabricius? & M. Nonius Balbus en Crète \\
\hline Janv. 27 av. J.-C. & \multicolumn{2}{|c|}{ Réforme augustéenne } \\
\hline Eté 27 av. J.-C. & A. Pupius Rufus ${ }^{61}$ & A. Pupius Rufus? \\
\hline
\end{tabular}

(57) Sur le statut de Lampa et de Kydonia: Dion Cassius, LI, 2, 3.

(58) Jones A.H. M., City, 1967, p. 129.

(59) SzRamkiewicz R., Gouverneurs, II, 1976, p. 84.

(60) Paribeni R., dans DE, II, 2, 1910, s. u. Creta, col. 1269-1271; malheureusement la liste proposée est alphabétique et non chronologique, et l'auteur ne donne aucune indication chronologique précise sur la période d'activité des gouverneurs; De LAET S., Senaat, 1941, p. 247 : les fastes sont plus complets, mais les gouvernements ne sont toujours pas précisément datés; cette liste est reprise par Eck W., Zephyrus, 23/24, 1972/73, p. 244-247; SzRamkiewicz R., Gouverneurs, II, 1976, p. 529-530: sa liste soulève des difficultés liées au fait qu'il rassemblait aussi bien les questeurs propréteurs que les gouverneurs de Crète et/ou de Cyrénaïque, sans tenir compte des modifications de 27 av. J.-C.; SANDERs I. F., Crete, 1982, p. 176-178: mais il ne précise pas si les gouverneurs ont eu en charge en plus de la Crète, la Cyrénaïque; Baldwin M. A., Fasti, 1983, p. 251; Thomasson B. E., Laterculi, 1984, col. 361-369; BALdwin-Bowsky M. A., CJ, 82, 1987, p. 223; LARONDE A., ANRW, II, 10, 1, 1988, p. 1015: mais il ne parle pas de la période située entre 31 et 27 av. J.-C.; RPC, I, p. 217-243: ils se fondent uniquement sur les attestations numismatiques; PAUTAsso A., ASAA, 72/73, 1994/95, p. 93-102 pour les fastes, en particulier p. 93-94: mais elle ne prend pas en compte les travaux les plus récents ni la totalité des informations fournies par les sources tant littéraires qu'épigraphiques, en particulier en ce qui concerne le patronat provincial de M. Nonius Balbus; Rémy B., Karthago, 24, 1999, p. 184: mais il ne propose pas de fastes pour la période qui nous intéresse, soit entre 31 et 27 av. J.-C.; la plupart des datations qu'il indique se bornent à mentionner «Auguste»; THOMAsson B. E., Laterculi, 2009, p. $151-152$.

(61) Guarducci M., Bulletino comunale di Roma, 56, 1928, p. 275-283 (AE, 1930, 62) = I. Cret, IV, 293 Gortyne:

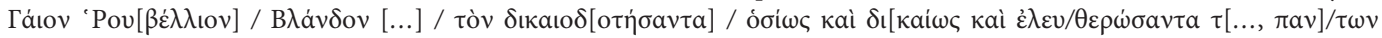

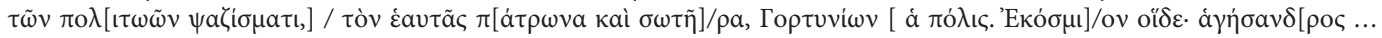

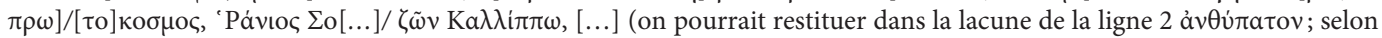
l'AE, il aurait été propréteur en Crète vers 30 av. J.-C.); CIL, III, 10 (AE, 1960, 266) Cyrène: C(aius) Rubelliu[s] Blan[dus], $\operatorname{proc}(o n) s(u l)$, porticus C[aes] ar[ei reficiendas curauit et dedicauit], / [...]lius $M($ arcu)f(ilius) Pacilarus, leg(atus) p[ropraetore] (cf. Gasperini L., QAL, 6, 1971, p. 7-9, C2); CIL, XIV, 3555 (ILS, 3401) Tibur; CIL, XIV, 3556 (ILS, 3098) Tibur. Cf. RE, I A, 1, 1914, s. u. Rubellius, col. 1158, n 5; Weidemann U., Acta Classica, 7, 1964, p. 64- 69; Eck W., Zephyrus, 23/24, 1972/73, p. 246-247; Szramkiewicz R., Gouverneurs, I, 1975, note 27; Thomasson B. E., Laterculi, 1984, col. 362, nº 8; Rémy B., Karthago, 24, 1999, p. 172, n²1; p. 182, n 56; p. 184; PIR², R, 109: proconsul Cretae et Cyrenarum.

(62) Falbe C.T., Lindberg J.C. et Müller L., Numismatique, 1860 p. 165-166, nº 429: il serait le premier proconsul de la province augustéenne. Cf. PARIBEnI R., dans DE, II, 2, 1910, s. u. Creta, col. 1270; PIR ${ }^{2}$, F, 85 : «temporibus Augusti»; SzRamkiewicz R., Gouverneurs, I, 1975, p. 25, note 27.

(63) $P_{I}^{2}$, P, 1088. Mais il ne semble avoir rempli de fonction qu'en Cyrénaïque, entre 30 et 27 av. J.-C. (Falbe C. T., Lindberg J.C. et MÜller L., Numismatique, 1860, p. 161-165, nº422-428; BMC Cyr., p. ccxxii-ccxxiii; Grant M., Imperium, 1946, p. 69; Chapman A., Num. Chron., 8, 1968, p. 23; Perl G., Klio, 52, 1970, p. 349-351, nº 19; Price M., INJ, 6/7, 1982/83, p. 121) bien que certains pensent plutôt à la période allant de 27 à 23 av. J.-C. (SzRAmkiewicz R., Gouverneurs, I, 1975, p. 25, note 27). Enfin T. V. Buttrey (dans Studies in Numismatic Method presented to Philip Grierson, 1983, p. 26-27; 31-32 et dans The Coinage of the Roman World in the Late Republic, 1987, p. 169) préfère le dater de la période pré-antonine. Voir aussi RPC, I, p. 221. 
Plusieurs propositions doivent être expliquées; nous avons ainsi préféré raccourcir le mandat de L. Pinarius Scarpus, daté par R. Szramkiewicz ${ }^{64}$ de 30 à 24 av. J.-C. Petit-neveu et héritier de César ${ }^{65}$, très proche du jeune Octavien depuis 44 av. J.-C., L. Pinarius Scarpus ${ }^{66}$ rejoint néanmoins Marc Antoine lors de la guerre de Pérouse ${ }^{67}$ et est ensuite nommé par ce dernier à un poste officiel en Cyrénaïque, peu de temps avant la bataille d'Actium. Durant son mandat ${ }^{68}$, il fait frapper des monnaies qui attestent de sa présence ${ }^{69}$.

Mais surtout, en 31 av. J.-C., il s'oppose à son protecteur, Marc Antoine ${ }^{70}$, et mettant à mort les émissaires du triumvir, refuse de lui apporter son aide, et de lui fournir ses quatre légions, alors que ce dernier débarque en 30 av. J.-C. sur les côtes libyennes ${ }^{71}$; il semble que sa reddition eut lieu à la fin de $31 \mathrm{av}$. J.-C. ${ }^{72} \mathrm{Il}$ reste donc en Cyrénaïque et aurait sans doute eu aussi des responsabilités en Crète dans les mois suivant la mort de Marc Antoine - M.A. Baldwin évoque "a special position $^{73}$ ", et «a special military command ${ }^{74}$ " pour l'année 29 av. J.-C. -; pendant les derniers mois de la lutte contre le triumvir vaincu, L. Pinarius Scarpus confie ses troupes ${ }^{75}$ à C. Cornelius Gallus $^{76}$, lieutenant d'Octavien et premier préfet d'Égypte. L'incertitude quant aux dates précises de la fin du mandat de L. Pinarius Scarpus rend difficile la datation de ses successeurs. Mais, quoi qu'il en soit, même s'il a duré un peu plus longtemps ${ }^{77}$, son mandat, de nature militaire, n'a pas pu aller au-delà de 27 av. J.-C.En effet, sa période d'activité post-antonienne est connue par des monnaies, datées de la période 30/27 av. J.-C.

(64) Szramkiewicz R., Gouverneurs, II, 1976, p. 529-530.

(65) Appien, BC, III, 22; Suétone, Iul., 83, 3. Cf. Münzer F., Hermes, 71, 1936, p. 229, note 2; Syme R., Aristocracy, 1986, table 3.

(66) Perl G., Klio, 52, 1970, p. 352-353, n²1; ID., Klio, 53, 1971, p. 378; Baldwin M.A., Fasti, 1983, p. 81-83, nº 1; PIR 2 , P, 413; Malitz J., Gymnasium, 111, 2004, p. 384.

(67) Appien, $B C$, IV, 107.

(68) Perl G., Klio, 52, 1970, p. 352-353, n²1; ID., Klio, 53, 1971, p. 378 : il n’aurait pas rempli un gouvernorat, mais simplement un commandement spécial.

(69) Sur les émissions monétaires de L. Pinarius Scarpus, cf. Crawford M., JRS, 64, 1974, p. 247; Gurval R. A., Actium, 1995, p. 51, note 62. Cf. annexe 3. Les monnaies de L. Pinarius Scarpus, doc. 1; 2.

(70) Bowersock G., Augustus, 1965, p. 27; Crawford M., JRS, 64, 1974, p. 247; Baldwin M. A., Fasti, 1983 , p. 5-6.

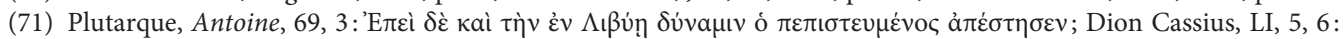

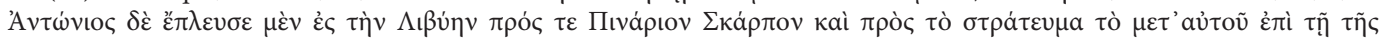

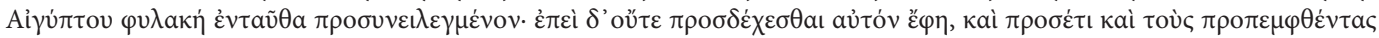

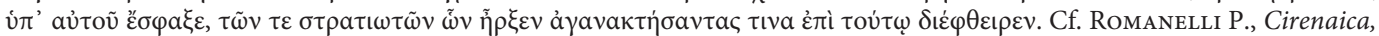
1943, p. 63-64; Tarn W.W. et Charlesworth M.P., CAH, X, 1963, p. 106; Chamoux Fr. Marc Antoine, 1986, p. 364; Pelling C., $C A H^{2}$, X, 1996, p. 59.

(72) Freyburger M.-L. et Roddaz J.-M., Dion Cassius, 50-51, 1991, p. 134, note 77. Auguste se vante d'avoir recouvré la province de Cyrénaïque dans son testament $(R G D A, 31,3$; cf. note 55$)$. L'ère provinciale débute d'ailleurs à l'automne 31, en souvenir de la bataille d'Actium; cf. Romanelli P., Cirenaica, 1943, p. 64; LARonde A., ANRW, II, 10, 1, 1988, p. 1015; Reynolds J. et Llyod J. A., CAH², X, 1996, p. 631.

(73) Baldwin M. A., Fasti, 1983, p. 82.

(74) Baldwin M. A., Fasti, 1983, p. 81.

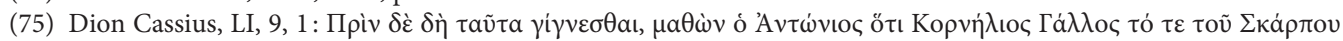

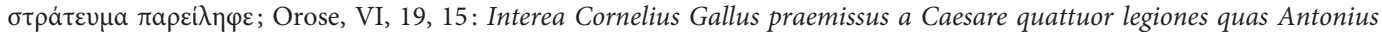
apud Cyrenas praesidii loco constituerat suscepit.

(76) PIR², C, 1369; Syme R., Aristocracy, 1986, p. 7; Reynolds J. et Llyod J. A., CAH², X, 1996, p. 631. Il est le premier préfet d'Égypte (cf. BAstianini G., ZPE, 17, 3, 1975, p. 267; ID., ZPE, 38, 1980, p. 75; ID., ANRW, II, 10, 1, 1988, p. 503; Bureth P., ANRW, II, 10, 1, 1988, p. 472).

(77) Orose, VI, 19, 15. Cf. Babelon E., Monnaies, II, 1885, p. 305; BMC Rep., II, p. 582 : il reste gouverneur jusqu'en 27 av. J.-C.; Romanelli P., Cirenaica, 1943, p. 63-64, note 2. Des monnaies avec la mention d'Octavien ont eu le temps d'être frappées; cf. Reynolds J. et Llyod J. A., CAH², X, 1996, p. 631. 
L'une d'elles en particulier est difficile à dater ${ }^{78}$. Afin d'y parvenir, il faut prendre en compte la date de la frappe des deniers octaviens avec ce type de revers imité sur les monnaies de L. Pinarius Scarpus; si le type est antérieur à Actium, ce dernier peut n'être resté en Cyrénaïque que jusqu'en 31 av. J.-C.; s'il est datable de 29 av. J.-C., il peut être resté quelques années de plus, jusqu'en 27 av. J.-C. par exemple ${ }^{79}$.

Certains types $^{80}$ sont à mettre en relation avec la victoire d'Octavien à Actium, et la main ouverte est «le symbole parlant du nom de Scarpus ${ }^{81}$ » mais on pourrait également y voir la volonté de L. Pinarius Scarpus de se tourner vers Octavien dont il reprend dans les légendes monétaires la propagande dynastique. Cette série devrait être placée très tôt après Actium, au plus tard dans les premiers mois de 30 av. J.-C.. Plus tard aurait été frappé un type portant le titre Augustus:

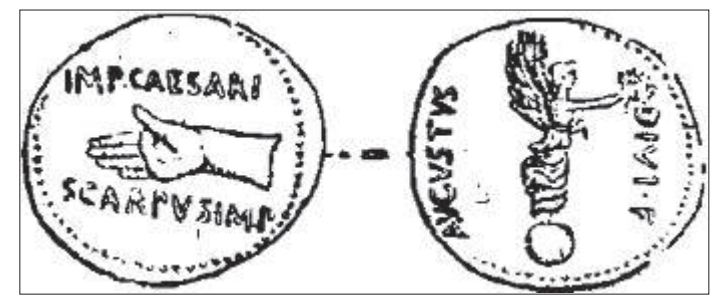

Fig. 1 :. La monnaie fictive de L. Pinarius Scarpus. Dessin tiré de Babelon E., Monnaies, II, 1885, p. 307, $\mathrm{n}^{\circ} 15^{82}$. Description: Avec la légende de droit IMP(eratori) CAESARI/ SCARPVs IMP(erator) associée à une main ouverte. La légende de revers AVGVstvs / DIVI F(ilius) accompagnant une Victoire à droite, debout sur un globe, tenant une couronne et une palme.

Mais, E. S. G. Robinson ${ }^{83}$ était très dubitatif quant à son existence; selon H. A. Grueber ${ }^{84}$ «this denarius ... described and illustrated ... but no specimen has been met with", et on trouve encore «the authenticity of this coin is doubtful ${ }^{85}{ }$; ce type n'est pas repris par M. Crawford ${ }^{86}$, et la fiabilité de cette source est mise en cause ${ }^{87}$. De plus, on n'entend plus parler de ce sénateur dans les sources après 30 av. J.-C. ${ }^{88}$. Il faut éliminer cette monnaie connue uniquement par un dessin, suivant en cela M. Crawford ${ }^{89}$ et K. $\mathrm{Kraft}^{90}$ : L. Pinarius Scarpus n'est donc pas resté en Cyrénaïque au-delà de 27 av. J.-C., alors qu'Octavien était devenu Augustus.

(78) Cf. annexe 3. Les monnaies de L. Pinarius Scarpus, doc. 3.

(79) RPC, I, p. 221.

(80) Cf. annexe 3. Les monnaies de L. Pinarius Scarpus, doc. $4 ; 5 ; 6$.

(81) Babelon E., Monnaies, II, 1885, p. 306.

(82) Cohen H., Médailles, I, 1880, p. 136, nº 497 = Babelon E., Monnaies, II, 1885 , p. $307, \mathrm{n}^{\circ} 15=$ p. $68, \mathrm{n}^{\circ} 165=B M C$ Rep., II, p. 582; 586 = RIC, I, p. 44, = BMC Emp., I, p. 111, nº69, pl. 17, n 16; Sydenham E. A., Coinage, 1952, p. 201, $\mathrm{n}^{\circ} 1285$ (pas de planche).

(83) BMC Cyr., p. ccx.

(84) BMC Rep., II, p. 586, note 1.

(85) RIC, I, p. 44.

(86) RRC, p. 542-543, nº 546.

(87) Perl G., Klio, 52, 1970, p. 352, note 7; Buttrey T.V., dans Studies in Numismatic Method presented to Philip Grierson, 1983, p. 32; $P I R^{2}$, P, 413 en particulier p. 165: «fide autem non dignum».

(88) Bowersock G., Augustus, 1965, p. 27.

(89) Crawford M., Hoards, 1969, p. 41, note 8-b; ID., JRS, 64, 1974, p. 247 : «the supposed issue for Octavian as Augustus is a fiction».

(90) Kraft K., Münzprägung, 1969, p. 223-224 (= Kleine Schriften, II, 1978, p. 309). 
Lui a sans doute succédé dans cette province, à une date qu'il faut placer entre 30 et 27 av. J.-C., A. Pupius Rufus ${ }^{91}$, connu par une monnaie ${ }^{92}$ dont le type n'a pas été retrouvé en Crète, ce qui laisserait supposer qu'il n'en était pas le gouverneur ${ }^{93}$. C'est donc sans doute au cours de l'été 27 av. J.-C. ${ }^{94}$, à la fin de son mandat "augustéen», que L. Pinarius Scarpus aurait laissé la place à A. Pupius Rufus ${ }^{95}$. Entre 31 et 27 av. J.-C., les fastes de la Cyrénaïque sont donc complets. M. Nonius Balbus ne peut donc avoir été proconsul de cette province avant 27 av. J.-C.; or, M. A. Baldwin considère qu'il a été le gouverneur de la double province, ce qui la conduit à placer son mandat après 27 av. J.-C. - date de la réunification des deux provinces que l'on place en 27 av. J.-C. ${ }^{96}$-, en 25 av. J.-C. ${ }^{97}$ par exemple. Mais ses arguments ne sont pas extrêmement convaincants; en effet, les problèmes liés aux territoires de Cnossos et de Gortyne qu'elle évoque ${ }^{98}$, s'ils ont effectivement été réglés par $\mathrm{M}$. Nonius Balbus ${ }^{99}$, ont pu l'être tout aussi bien avant qu'après $27 \mathrm{av}$. J.-C. Elle lie en effet la fondation de la colonie de Cnossos, l'octroi de terres à Capoue ${ }^{100}$ et un conflit avec Gortyne à la présence de M. Nonius Balbus ${ }^{101}$, ce qui la conduit à placer l'octroi des terres à Capoue au mieux en 27 av. J.-C. ${ }^{102}$ ! Elle pense que l'origine campanienne de M. Nonius Balbus a rassuré les Capouans qui auraient ainsi vu leurs intérêts confiés à des mains amicales ${ }^{103}$. Mais les dédicaces offertes par les deux cités de Cnossos et de Gortyne au sénateur font visiblement partie d'une série où le commune Cretensium est trois fois représenté. Il semble donc que c'est dans un autre contexte, légèrement antérieur à la réorganisation administrative de 27 av. J.-C., qu'il faille placer le proconsulat de M. Nonius Balbus. Ayant fait preuve de sa loyauté en 32 av. J.-C., lors de son tribunat de la plèbe, il est donc normal qu'après sa préture, remplie peut-être en 30 av. J.-C. ${ }^{104}$, Octavien ait pensé à

(91) Cf. ci-dessus, p. 255.

(92) $B M C$ Cyr., p. ccviii (pour lui, il ne peut pas avoir été gouverneur après 27 av. J.-C.); p. 117-119, $\mathrm{n}^{\circ} 27-35=R P C, \mathrm{I}$, p. 221-222, n 919-923 (cf. annexe 4. Monnaies mentionnant des gouverneurs de Crète ou de Cyrénaïque, doc. 2).

(93) BMC Cyr., p. ccix; SANDERS I. F., Crete, 1982, p. 6, note 40; 176: «it is possible that his governorship may have been there (Cyrene) only».

(94) C’est une possibilité évoquée par la $P I R^{2}, P$, p. 165.

(95) Mais pour certains (RPC, I, p. 221), A. Pupius Rufus serait à placer avant L. Pinarius Scarpus, entre 34 et 31 av. J.-C.

(96) Romanelli P., Cirenaica, 1943, p. 69-70; Sartre M., Orient, 1991, p. 22: mais la date de reconstitution d'une seule province est simplement effleurée: «Recréée (la province bicéphale) au lendemain de l'occupation de l'Égypte»; Reynolds J. et Llyod J.A., CAH², X, 1996, p. 631: «At any rate from 27 B. C. Cyrenaica was administered together with Crete»; Levick B. M., CAH², X, 1996, p. 648; RÉmy B., Karthago, 24, 1999, p. 165-166.

(97) La date de «vers 25 av. J.-C.» est proposée par M.A. BAldwin (Fasti, 1983, p. 83-88; 251; CJ, 82, 1986, p. 220: "one of the earliest governors of the Augustan double province»), suivie par A.-M. RouAnet-Liesenfelt (Ktèma, 19, 1994, p. 10). R. SzRAmkiewicz (Gouverneurs, II, 1976, p. 530) donne une date indéterminée entre 22 av. J.-C. et 14 ap. J.-C.

(98) Baldwin-Bowsky M.A., CJ, 82, 1987, p. 218-229.

(99) Baldwin-Bowsky M. A., CJ, 82, 1987, p. 220: «The Herculaneum inscriptions to Balbus testify to his crucial role in Augustan territorial settlements in central Crete». Mais les textes ne parlent pas de ce sujet.

(100) Selon K. J. Rigsby (TAPA, 106, 1976, p. 328-330) suivi par M. A. BAldwin-Bowsky (CJ, 82, 1987, p. 220). F. Imhoof-Blumer (Monnaies, 1883, p. 212) pensait que la colonie aurait été fondée avant la bataille d'Actium et, que l'octroi des terres à Capoue aurait eu lieu, selon lui, entre 31 et 27 av. J.-C.. Mais rien n'oblige à lier l'octroi des terres et la fondation coloniale; cf. PerL G., Klio, 52, 1970, p. 343.

(101) BALdwin-Bowsky M.A., CJ, 82, 1987, p. 220.

(102) Elle s'appuie pour cela (BALDwin-Bowsky M. A., CJ, 82, 1987, p. 223) sur l'idée de K. J. Rigsby (TAPA, 106, 1976, p. 328-330) proposant de placer l'assignation effective des terres de Cnossos à Capoue entre 36 et 27 av. J.-C., et plutôt vers 27 av. J.-C.

(103) BALdwin-Bowsky M. A., CJ, 82, 1987, p. 224-225. Mais cela ne permet pas alors de comprendre pourquoi ce sont les Crétois qui l'ont choisi comme patron! On se serait attendu à des hommages provenant de la cité de Capoue si M. Nonius Balbus était réellement intervenu en leur faveur lors de l'assignation des terres de Cnossos. Elle est suivie par A. PAUTAsso (ASAA, 72/73, 1994/95, p. 77).

(104) BALdwin-Bowsky M. A., CJ, 82, 1987, p. 223. 
ce jeune sénateur ${ }^{105}$ pour ce poste. M.A. Baldwin estime que son proconsulat ne peut alors pas se placer avant $26 / 25$, voire même $25 / 24$ av. J.-C. ${ }^{106}$, ce qui permettrait de respecter les intervalles normaux du cursus honorum. Mais il convient de se demander si, dans les années qui ont suivi Actium, le nombre des sénateurs fidèles et capables était suffisant pour reprendre en main non seulement l'Orient antonien mais aussi les nouveaux cadres administratifs envisagés par Octavien. M.A. Baldwin-Bowsky, pour confirmer les «coïncidences» présentées, évoque la possibilité de manipuler le tirage au sort annuel ${ }^{107}$. Pourquoi M. Nonius Balbus n'aurait-il pas bénéficié de cette pratique pour être envoyé en Crète dès 29 ou 28 av. J.-C. ${ }^{108}$ ?

C'est donc uniquement à la Crète ${ }^{109}$ que doit être, à notre avis, rattaché M. Nonius Balbus, ce qui explique sans doute l'absence de la mention des Cyrénaïcains dans les hommages qui lui sont rendus à Herculanum. Ce sénateur a donc eu pour tâche de replacer la province de Crète dans un cadre provincial et d'éliminer toute velléité d'autonomie et toute trace du parti antonien. Face à cette tutelle plus lourde que ce qu'ils supportaient dans les années précédentes, les Crétois n'ont pu que se sentir inquiets. Néanmoins, la politique menée par le sénateur, et l'attitude finalement favorable de l'île envers Octavien a sans doute permis que la Crète soit considérée comme une province tranquille, pacifiée et sans risque; elle est ainsi attribuée, lors du partage de 27 av. J.-C., au Sénat et au peuple romain. Mais c'est au cours de l'été 27 que, les mandats des gouverneurs en place lors de la réforme en janvier étant terminés, le premier gouverneur unique a été nommé. Il s'agirait sans doute d'A. Pupius Rufus, même si les indices de cette nouvelle situation sont rares. Par la suite, cette double province a été confiée à des sénateurs de rang prétorien, à la carrière relativement modeste ${ }^{110}$.

Les provinciaux ont été soumis à une tension forte, à une inquiétude liée à l'émergence du pouvoir augustéen, à la reprise en main des provinces, à la modification de leur statut. Comment alors faire part de leurs peurs à un Prince lointain, et dont ils ont été les adversaires?

\section{NONIUS BALBUS DÉSIGNÉ PAR LE KOINON}

C'est par la désignation d'un patron provincial que le contact a pu être établi. Ce dernier est fort logiquement nommé par le koinon local. Dans le cas particulier de la Crète, le koinon est attesté dès l'époque hellénistique ${ }^{111}$, sa création remontant sans doute à la fin

(105) Sa jeunesse sur les portraits le représentant à Herculanum, qui sont postérieurs à son gouvernement crétois, est souvent évoquée. Cf. Adamo Muscettola S., Prospettiva, 28, 1982, p. 4.

(106) Baldwin-Bowsky M. A., CJ, 82, 1987, p. 223.

(107) BALdwin-Bowsky M.A., CJ, 82, 1987, p. 224: «The annual lottery was known to be subject to adjustement, to manipulation, and also to outright suspension when special circumstances in the province could be foreseen to warrant special attention». Cf. TAlbert R. J. A., Senate, 1984, p. 347-353; 397.

(108) Gardthausen V., Augustus, 1891/1904, II, p. 178, $\mathrm{n}^{\circ}$ 16: «Die Inschriften von Cretensern gesetzt sind und Cyrene nicht erwähnt wird, so war Balbus vielleicht vor dem Jahre 727/27 Statthalter, ehe Creta und Cyrene vereinigt wurden".

(109) Il est pourtant très régulièrement mentionné comme proconsul de Crète et de Cyrénaïque; cf. par exemple Adamo Muscettola S., Prospettiva, 28, 1982, p. 2.

(110) Laronde A., ANRW, II, 10, 1, 1988, p. 1016: «Dans l'ensemble, les proconsuls de Crète et de Cyrénaïque font des carrières solides, mais non brillantes »; RÉmy B., Karthago, 24, 1999, p. 166.

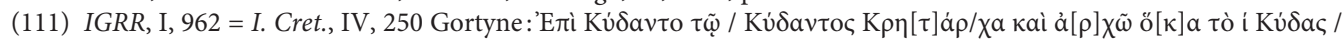

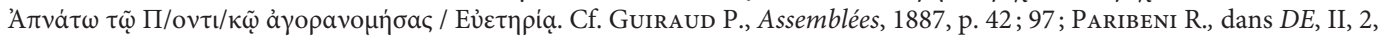
1910, s. u. Creta, col. 1260; Muttelsee M., Kretas, 1925, p. 39-43; Van der Mijnsbrugge M., Koinon, 1931; Raven E. J. P., Num. Chron., 18, 1938, p. 151; van Effenterre H., Crète, 1948, p. 127-160; 213-220 sur le koinon et la politique étrangère de la Crète à l'époque hellénistique; Perrot G. dans Daremberg C. et Saglio E., Dictionnaire, I-2, 1887, s. u. Cretarchia, p. 1562-1563; Caillemer E. dans Daremberg C. et Saglio E., Dictionnaire, I-2, 1887, s. u. Cretensium respublica, 
du III $^{\text {e }}$ siècle av. J.-C. ${ }^{112}$, dans le cadre des conflits internes aux cités crétoises ${ }^{113}$. Mais, il ne comprend pas toutes les cités de l'île, ce qui ne permet pas de totalement l'assimiler avec les concilia / koina provinciaux d'époque impériale. Bien attesté dans l'épigraphie, il est donc, avant la conquête romaine, davantage une manifestation de l'hégémonie de telle ou telle cité - Gortyne et Cnossos principalement -, sans que l'on sache à quelle fin il était utilisé, ni s'il avait un réel pouvoir face aux étrangers ${ }^{114}$-on peut signaler que, lors des rencontres diplomatiques ${ }^{115}$, c'est avec les Crétois organisés en koinon que les Romains traitent ${ }^{116}$. En 71/70 av. J.-C., les Crétois envoient à Rome une ambassade constituée de trente représentants pris parmi les plus brillants personnages de l'île ${ }^{117}$, et chargés de manifester leur bonne foi, et, Pompée ${ }^{118}$ reçoit en 67 av. J.-C. une délégation du commune Cretensium ${ }^{119}$ - le terme est employé par Cicéron ${ }^{120}$ - constituée des représentants de plusieurs cités crétoises, et envoie un légat chargé d'obtenir la soumission des Crétois; il semble donc, qu'à cette époque, cette assemblée soit davantage reconnue et puisse jouer un rôle politique fédérateur ${ }^{121}$; elle aurait été restaurée par Q. Caecilius Metellus Creticus en 67 av. J.-C. ${ }^{122}$; mais les informations sur les décennies suivantes sont relativement modestes ${ }^{123}$.

p. 1563-1568, en particulier p. 1565-1566; Deininger J., Provinziallandtage, 1965, p. 12; Willets R. F., Kadmos, 1975, p. 143-148; Brulé P., Piraterie, 1978, p. 33-34; 43, note 1; 81-88 (sur l'asylie); Bertrand J.-M., dans Rome et la conquête du monde méditerranéen, 2, 1978, p. 814; Rouanet-Liesenfelt A.-M., Ktèma, 19, 1994, p. 7; Haensch R., Capita, 1996, p. 206.

(112) Sur la mise en place des alliances successives des cités crétoises, cf. Strabon, X, 4, 7; 11. Sur les conflits fréquents en Crète et le rôle des alliances, cf. Plutarque, De frat. amore, $19 \mathrm{~B}$.

(113) On peut citer par exemple le conflit opposant Cnossos et Gortyne à la cité de Lyttos en 221/220 av. J.-C. (Polybe, IV, 53, 4). Cf. VAn der Mijnsbrugge M., Koinon, 1931, p. 13; 58 et s.

(114) Rouanet-Liesenfelt A.-M., Ktèma, 19, 1994, p. 8.

(115) VAn Der Mijnsbrugge M., Koinon, 1931, p. 27-34: «The foreign relations of the cretan koinon».

(116) Tite Live, XXXVII, 60 en 189 av. J.-C.; Polybe, XXII, 15 en 184; Tite Live, XLIII, 7 en 170 (cf. Jal P., Tite Live, XXXII, 1976, p. 107, note 1: les légats envoyés auprès des Romains le sont par «certaines villes crétoises isolées»). Cf. Willets R. F., Society, 1955, p. 238-240.

(117) Diodore de Sicile, XL, 1, 1 ; Dion Cassius, XXX-XXXV, 111; Pseudo-Asconius, In diu., p. 202, \$55.

(118) Plutarque, Pompée, 29, 4; Dion Cassius, XXXVI, 18-19; Cicéron, De imp. Cn. Pompei, 12, 35; 16, 46; Florus, I, 42, 5 ; Eutrope, VI, 11, 2; Orose, VI, 4, 2; HeAD B.V., $H N^{2}$, 1963, p. 467-468: il s'agit d'un type portant au revers ГOPTYN et en particulier une tête d'éléphant, emblème de la gens Caecilia Metella; la monnaie peut être mise en rapport avec la conquête de la Crète par Q. Caecilius Metellus Creticus.

(119) Cicéron, De imp. Cn. Pompei, 12, 35; 16, 46. Il semble que ,dès les négociations avec Rome en 70 av. J.-C., le koinon ait joué un rôle non négligeable. Cf. Willets R.F., Kadmos, 14, 1975, p. 145.

(120) Cicéron emploie également le terme commune pour désigner l'assemblée provinciale des Siciliens: Cicéron, II Verr., 2, XXXVII, 90: quam non istam communem Siculorum tempestatem calamitatemque uitaret; XLII, 103: Dicit praetera testimonium tota Sicilia, quae in communibus postulatis ciuitatum omnium consulibus edidit; XLVI, 114: cui statua Romae stant inauratae a communi Siciliae, quem ad modum inscriptum uidemus; LIX, 145: ut idem pro parte in commune Siciliae conferrent; LIX, 146: in hanc rem testem totam Siciliam citabo, quae mihi una uoce statuarum nomine magnam pecuniam per uim coactam esse demonstrat. Nam legationes omnium ciuitatum in postulatis communibus.; LXIII, 154: Itaque eum non solum PATRONVM illius insulae, sed etiam $\Sigma \Omega$ THPA inscriptum uidi Syracusis ... huic etiam Romae uidemus in basi statuarum maximis litteris incisum, a commune Siciliae datas; LXIX, 168: cum nideret statuas ab negotiatoribus, ab aratoribus, a communi Siciliae positas?. Cf. SARTORI F, Klio, 63, 1981, 2, p. 402 . Il emploie également le terme pour désigner la confédération des Milyades: Cicéron, II Verr., 1, XXXVIII, 95: Pro quaestore uero quo modo iste commune Myliadum uexarit; 96: QUOD a COMMUNi Milyadum. D'autres auteurs ont recours à ce mot pour désigner une confédération, une association ou un ensemble de peuples ou de cités; cf. par exemple, Ovide, Métamorphoses, XII, 1: mille rates gentisque simul commune Pelasgae (évocation de la conjuration grecque après l'enlèvement d'Hélène).

(121) Luzzatto G. I., Roma, 1985, p. 156: «Paradossalmente la conquista romana, che trasporta la sede del koเvóv a Gortina e lo adatta a concilium prouinciae per le consuete manifestazioni di lealismo dinastico, sembra ravvivare l'istituzione».

(122) VAn Der Mijnsbrugge M., Koinon, 1931, p. 71; l'auteur signale qu'il y aurait eu des modifications dans

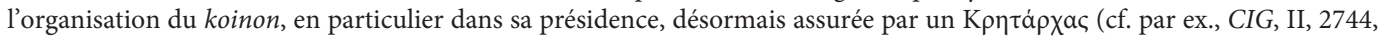

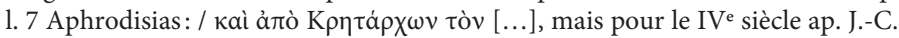

(123) Paluchowski A., Elites, 2004, p. 203. 
En 43 av. J.-C., Marc Antoine restructure ${ }^{124}$ le koinon et l'assemblée, si elle garde le nom de

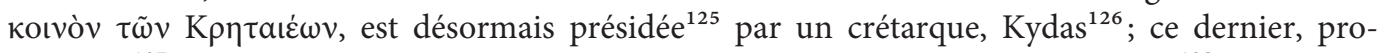
antonien $^{127}$, dispose d'un pouvoir de nature sans doute militaire sur les Crétois ${ }^{128}$, désormais libres, assumant la responsabilité du territoire comme un gouverneur de province le ferait ${ }^{129}$, pendant la durée du séjour de Marc Antoine en Orient ${ }^{130}$. La présence du koinon est plus manifeste: désormais, il dispose d'un monnayage mentionnant le nom de $\mathrm{Kydas}^{131}$ et sa fonction, crétarque ${ }^{132}$, que certains interprètent comme étant «in fact a Roman provincial issue, as Roman as the similar coinages in the province of Asia signed a few years previously by the provincial governors ${ }^{133}$ ». On doit supposer que les Crétois étaient alors particulièrement favorables à Marc Antoine, qui leur aurait laissé une certaine autonomie, même si ce dernier inclut des domaines crétois dans les territoires accordés à Cléopâtre et à leurs enfants ${ }^{134}$ en 37/36 av. J.-C., et, si des légats sont régulièrement envoyés ${ }^{135}$ dans la région entre 43 et 37 av. J.-C. Selon M.A. Baldwin ${ }^{136}$, c'est à partir de cette dernière date, que les Crétois auraient bénéficié d'une plus grande liberté sous l'autorité de $\operatorname{Kydas}^{137}$, agent antonien, alors que l'île relève en partie de l'autorité de la reine égyptienne.

Néanmoins, l'influence octavienne avait semblé présente dans l'île, en sus de la diminution du territoire de Cnossos, dont une partie a été attribuée à la cité de Capoue ${ }^{138}$, comme en témoignerait

(124) Jones A.H.M., City, 1967, p. 117; Sanders I.F., Crete, 1982, p. 5-6; Rouanet-Liesenfelt A.-M., dans Aux origines de l'hellénisme, la Crète et la Grèce, 1984, p. 343-352; EAD., Ktèma, 19, 1994, p. 8.

(125) Il faut rester d'une grande prudence sur la fonction de crétarque comme le souligne J. DeININGER (Provinziallantage, 1965, p. 12; 43; 84-85), suivi par A.-M. RouAnet-Liesenfelt (dans Aux origines de l'hellénisme, la Crète et la Grèce, 1984, p. 346-347.

(126) IGRR, I, 962 = I. Cret., IV, 250 Gortyne (cf. note 110). Cf. PARIBEni R., dans DE, II, 2, 1910, s. u. Creta, col. 1261; Sanders I.F., Crete, 1982, p. 6; Rouanet-Liesenfelt A.-M., dans Aux origines de l'hellénisme, la Crète et la Grèce, 1984, p. 343-352; RPC, I, p. 222; PALuchowski A., Elites, 2004, p. 203 : il rassemble tous les témoignages portant sur ce personnage.

(127) Cicéron, Phil., V, 13: Cydam Cretensem, portentum insulae, hominem audacissimum et perditissimum Gortynium uero iudicem quis nouit aut quis nosse potuit?... Cretensis iudex isque nequissimus; VIII, 9, 27: An ne suorum aliquis a Cyda, Lysiade, Curio condemnetur?; VIII, 27: An ne suorum aliquis a Cyda, Lyside, Curio condemnetur? Cf. ImHoof-BLUmer F., Monnaies, 1883, p. 211, note 7 (le portrait dressé du personnage est peu sympathique!); Chapman A., Num. Chron., 8, 1968, p. 21-22, note 5: «If Kydas was Antony's agent and appointed by Antony to manage Cretan affairs».

(128) Rouanet-Liesenfelt A.-M., Ktèma, 19, 1994, p. 9.

(129) Il semble que la Crète ne soit plus totalement une province en 44 av. J.-C.; cf. Cicéron, Phil., II, 38, 97 : statuiturque ne post M. Brutum pro consule sit Creta prouincia ... An Caesaris decreto Creta post M. Bruti decessum potuit liberari, cum Creta nihil ad Brutum Caesare uiuo pertineret? At huius uenditione decreti, ne nihil actum putetis, prouinciam Cretam perdidistis. Mais quel est alors le rôle de Crassus? Cf. Chapman A., Num. Chron., 8, 1968, p. 21-22; Paluchowski A., Elites, 2004, p. 223.

(130) Il aurait été crétarque au moins dix ans; cf. Rounnet-Liesenfelt A.-M., dans Aux origines de l'hellénisme, la Crète et la Grèce, 1984, p. 351. G. PerL (Klio, 52, 1970, p. 344-346) date plus particulièrement son mandat des années 34/31 av. J.-C..

(131) Cf. annexe 5. Les monnaies de Kydas.

(132) Cf. annexe 5. Les monnaies de Kydas, doc. 2.

(133) Price M., INJ, 6/7, 1982/83, p. 120. Cette affirmation est reprise par T. V. Buttrey (dans The Coinage of the Roman World in the Late Republic, 1987, p. 167).

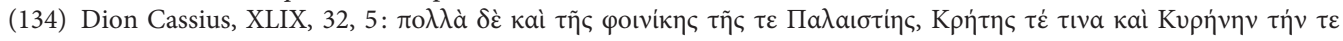

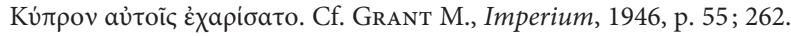

(135) Qu'ils soient envoyés par Marc Antoine comme un véritable gouverneur, ou par Cléopâtre; cf. CHAPMAN A., Num. Chron., 8, 1968, p. 16, note 1 sur le sujet.

(136) Baldwin M. A., Fasti, 1983, p. 4.

(137) Une partie des frappes monétaires de Kydas est à dater au plus tôt de 37 av. J.-C.; cf PALUCHOwsKi A., Elites, 2004, p. 206.

(138) Cf. ci-dessus, p. 176; 183. Pour certains, les deux événements sont à relier, et cela les conduit à faire de Cnossos une colonie de Capoue. Mais, dans les sources, les deux épisodes ne sont pas mentionnés conjointement; contre le fait que 
une monnaie émise par la colonie nouvellement fondée de $\operatorname{Cnossos}^{139}$ : si sur le droit figurerait la tête de Marc Antoine, sur le revers serait présente celle d'Octavien ${ }^{140}$. Mais cette datation précoce, 36 av. J.-C. ${ }^{141}$, de la fondation coloniale pose problème; en effet, il est difficile d'admettre qu'Octavien fonde une colonie dans un territoire placé sous l'influence de Marc Antoine et entre les mains de Cléopâtre ${ }^{142}$. Il faut sans doute déplacer cette fondation, et préférer une date postérieure à la bataille d'Actium, vers 30 av. J.-C. ${ }^{143}$, comme en témoigne son nom de Colonia Iulia Nobilis Cnossos - certains placeraient même sa fondation après 27 av. J.-C. ${ }^{144}$, mais elle se serait alors sans doute appelée colonia Augusta, comme les colonies fondées après cette date ${ }^{145}$. Les documents connus pour cette fondation peuvent très bien convenir pour cette datation post-triumvirale. Ainsi, la monnaie évoquée ci-dessus pourrait avec plus de vraisemblance représenter Auguste et Agrippa ${ }^{146}$, la titulature de la cité, C I N CN, étant identique à celle utilisée dans les émissions monétaires considérées comme postérieures. La cité dispose désormais d'une organisation municipale de type romain, dirigée par des duumuiri. Sont connus en particulier trois couples, T. Fufius et M. Aemilius, C. Petronius et M. Antonius, et, Aeschinus Caes. L iter et Plotius Plebeius ${ }^{147}$. De plus, subsistent jusqu'après Actium, des monnaies émises par les magistrats grecs de Cnossos ${ }^{148}$; trois émissions sont attestées postérieurement à la présence de Crassus en $37 / 36$ av. J.-C. ${ }^{149}$.

Cnossos soit une colonie de Capoue, cf. Perl G., Klio, 52, 1970, p. 343; ID., Klio, 53, 1971, p. 375; Rigsby K. J., TAPA, 106, 1976 , p. 323.

(139) En 36 av. J.-C. selon certains, suivant Dion Cassius, XLIX, 14. Cf. pour la première mention, mais sans donner de

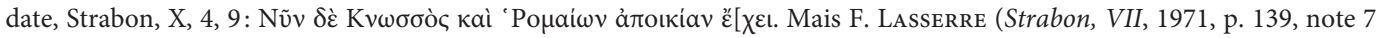
de la p. 89) donne la date de 36 av. J.-C. pour la fondation de la colonie.

(140) Imhoof-Blumer F., Monnaies, 1883, p. 212-213 (mais l'auteur indique qu'il pourrait aussi s'agir de Lépide et d'Octavien, si les pièces venaient d'Espagne, ce qui n'est pas le cas); Svoronos J., Numismatique, 1890, p. 89-90, n ${ }^{\circ} 180$ à 183, pl. VIII, $\mathrm{n}^{\circ} 11$ et 12 : Marc Antoine et Octavien (il est suivi par Grant M., Imperium, 1946, p. 262); BMC Cyr., p. ccix, note 2: il préfère voir Octavien et Agrippa, tout comme Chapman A., Num. Chron., 8, 1968, p. 14, note 1, et RPC, I, p. 234; p. 236, n 976, pl. 55, nº 976 (cf. annexe 6. Les magistrats de la colonie de Cnossos dans les monnaies augustéennes, doc. 1). Cf. Grant M., Imperium, 1946, p. 261-263 qui propose d'y voir Auguste et éventuelllement Tibère; VitTinghoff P., Kolonisation, 1951, p. 131, note 3; BALdwin M. A., Fasti, 1983, p. 5.

(141) M.A. BALdwin-Bowsky (CJ, 82, 1987, p. 220) associe la fondation coloniale et l'expropriation de terres en faveur de Capoue.

(142) Perl G., Klio, 52, 1970, p. 343, note 2.

(143) Levick B. M., $C A H^{2}$, X, 1996, p. 648 pense que la fondation de la colonie a eu lieu en 30 av. J.-C.; PerL G., Klio, 52,1970 , p. 343-344 estime que la fondation ne peut avoir eu lieu qu'avant 44 av. J.-C. ou plutôt après 31 av. J.-C., en raison de la présence de Marc Antoine; Sanders I. F., Crete, 1982, p. 5; 14; Pautasso A., ASAA, 72/73, 1994/95, p. 76. Pour le contexte des fondations coloniales après la bataille d'Actium, cf. SALmon E.T., Colonization, 1969, p. 138 et suivantes. I.E. Tzamtzis (dans Studi sull'età di Marco Antonio, 2006, p. 362) place la fondation "probablement en 27 ou plus tard, mais certainement après Actium».

(144) Deux émissions monétaires mentionnant AVG pourraient être les «real foundation issues» selon SANDERS I. F., Crete, 1982, p. 14. Cf. aussi Rigsby K. J., TAPA, 106, 1976, p. 324; 329: «the colony at Cnossus cannot be shown to exist before Augustus» (il placerait la fondation coloniale en 27 av. J.-C.; pour ses arguments, cf. p. 329-330); PRICE M., INJ, 6/7, 1982/83, p. 122: "there is no evidence that a colony had been founded at Cnossus in the late Republic and its foundation is probably to be placed after Octavian's acceptance of the title Augustus »; cf. annexe 6. Les magistrats de la colonie de Cnossos dans les monnaies augustéennes, doc. 4 .

(145) Comme par exemple la colonie d'Augusta Emerita en 25 av. J.-C.; cf. entre autres, Alvarez y SAEnz DE Buruaga J., dans Augusta Emerita, 1976, p. 19-32; ID., Museos, 1, 1982, p. 5-7; CANTo A. M., Gerión, 7, 1989, p. 149-20; EAD., dans Stadtbild und Ideologie, 1990, p. 289-297.

(146) Chapman A., Num. Chron., 8, 1968, p. 14, note 1, suivant BMC Cyr., p. CCIX, note 2; cf. aussi Perl G., Klio, 52, 1970, p. 344; SANDers I. F., Crete, 1982, p. 5; 14; Buttrey T. V., dans The Coinage of the Roman World in the Late Republic, 1987, p. 171. Sur les représentations d'Auguste accompagné d'Agrippa, cf. Hurlet F., Collègues, 1997, p. 406-411.

(147) Cf. annexe 6. Les magistrats de la colonie de Cnossos dans les monnaies augustéennes, doc. $1 ; 2 ; 3$.

(148) Chapman A., Num. Chron., 8, 1968, p. 13; Sanders I. F., Crete, 1982, p. 14.

(149) Chapman A., Num. Chron., 8, 1968, p. 16-17: Mnesitheos, Tharsydikas et Tauriadas. 
Quoi qu'il en soit, dans les mois qui ont suivi Actium, même si le koinon est dissous, si le titre de son président change en archiereus - on ne trouve désormais pas d'autre crétarque avant le $\mathrm{IV}^{\mathrm{e}}$ siècle dans un document peu clair ${ }^{150}$ - et, si de nouveaux délégués sont désignés par les cités membres, la structure continue à jouer son rôle d'intermédiaire - et se tourne peu à peu vers l'organisation du culte impérial provincial ${ }^{151}$-, et c'est donc tout naturellement elle qui désigne un patron provincial chargé de plaider sa cause à Rome.

Le patron choisi par les Crétois ne manque pas d'intérêt: M. Nonius Balbus est bien connu par de nombreuses inscriptions découvertes à Herculanum. Originaire de Nuceria ${ }^{152}$, ce tribun de la plèbe de 32 av. J.-C. intercède en faveur d'Octavien contre les propositions de C. Sossius ${ }^{153}$. Il a ensuite été préteur ${ }^{154}$, puis devient proconsul de Crète aux environs de 29/28 av. J.-C., sans que l'on sache à quel moment il quitta sa fonction; certains ont pu penser qu'il serait l'un des tous premiers, si ce n'est le premier gouverneur de la double province de Crète et Cyrénaïque réorganisée par Auguste en 27 av. J.-C.. Nous restons très réservée quant à cette hypothèse ${ }^{155}$. Son emploi du temps de gouverneur a dû être chargé: pour M.A. Baldwin ${ }^{156}$, il intervint, entre autres, dans les problèmes liés à la localisation des territoires de Cnossos, Gortyne et Capoue; on ne peut en effet nier le traumatisme engendré par toute fondation coloniale, lié au démembrement et au découpage des territoires, domaines et ressources, même si les Romains veillaient à maintenir des relations avec les populations déplacées ${ }^{157}$. Comme tout gouverneur, il a accordé la citoyenneté romaine à des Crétois, comme l'atteste l'onomastique de quelques personnages ${ }^{158}$.

On comprend que les Crétois le choisissent comme patron pour les représenter auprès d'Octavien $^{159}$ : proche du Prince qui lui a donné sa confiance, il retourne à Rome, où il siège au Sénat, sans doute au cours de l'été 28 av. J.-C.. C'est donc lors de son gouvernement ${ }^{160}$ ou plus sûrement à sa sortie de charge ${ }^{161}$, qu'il fut choisi comme patron par la province de Crète. Il connaît la province, ayant pu l'aider à passer les mois difficiles qui suivent la reprise en main, il a sans doute rendu service à certaines cités pro-octaviennes, et a dû tenir compte de la situation particulière de la Crète pour administrer une population qu'il fallait séduire. On peut se demander si des liens plus anciens unissaient la province avec la gens Nonia; en effet, un Nonius est peut être connu comme gouverneur de la Crète, ou peut-être des deux provinces, quand Cicéron gouverne la Cilicie ${ }^{162}$ en

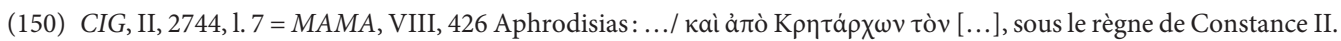

(151) Van der Mijnsbrugge M., Koinon, 1931, p. 71-72; Sanders I. F., Crete, 1982, p. 8-9.

(152) CIL, X, 1429(ILS, 896) Herculanum. Cf. Pflaum H.-G., Historia, 13, 1964,p. 120 ; Camodeca G., EOS, II, 1982 , p. 125.

(153) Dion Cassius, L, 2, 3.

(154) En 30 av. J.-C. pour Baldwin-Bowsky M. A., CJ, 82, 1987, p. 223.

(155) Cf. ci-dessus, la présentation des fastes de Crète et de Cyrénaïque, tableau 1.

(156) Baldwin M.A., Fasti, 1983, p. 49: «M. Nonius Balbus appears to have been involved in the original settlement of the territories of Cnossus, Gortyn and Capua in the Iraklion plain of Crete; for his efforts he received inscriptions in gratitude from the Cretans»; p. 87-88; BALDwin-Bowsky M.A., CJ, 82, 1987.

(157) Cf. par exemple le cas de la colonie d'Aventicum, Frei P., Pro Aventico, 20, 1969, p. 5-22.

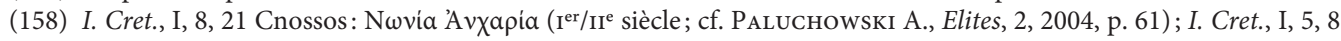

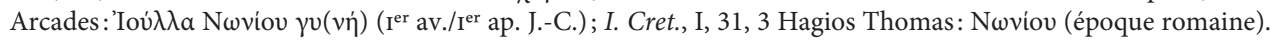

(159) Sanders I. F., Crete, 1982, p. 9: «Outside Crete, the Council maintained links with various men position who acted as its patron».

(160) Pour Nicols J., ZPE, 80, 1990, p. 101.

(161) Guiraud P., Assemblées, 1887, p. 181.

(162) Cicéron, Att., VI, 1, 13 (CCXlv) en février 50 av. J.-C.; ce sont les traducteurs, L.A. Constans et J. BAYeT (Cicéron, IV, 1962, p. 146, note 5) qui mentionnent ce gouvernement Cf. Hölzl M., Fasti, 1876, p. 69-70; SHACKLETON BAILEY D. R., Letters, III, 1968, p. 246: pour lui, il s'agit du gouverneur de Macédoine, qui n'aurait donc aucun lien avec la Crète; Paribeni R., dans DE, II, 2, 1910, s. u. Creta, col. 1270; Perl G., Klio, 52, 1970, p. 332, n 6. Sur le contexte du gouvernement de la Cilicie par Cicéron, cf. D’Hugues G., Proconsulat, 1876; Grimal P., Cicéron, 1986, p. 279-296. 
51/50 av. J.-C. Mais il ne s'agit que d'une hypothèse, ce personnage n'étant pas connu par ailleurs, même si certains l'assimilent à M. Nonius Sufenas ${ }^{163}$.

C'est donc le koinon qui coopte son patron ${ }^{164}$; il est malheureux que, sur les trois hommages qui lui sont rendus à Herculanum par la province, le terme de commune soit un terme restitué à deux reprises ${ }^{165}$. Cependant l'existence du troisième document ${ }^{166}$ rend convaincante cette restitution.

Nous ignorons comment il a rempli son rôle de patron provincial auprès du Prince, essayant sans doute d'éviter un sort trop dur à la province. Quoi qu'il en soit, son action a visiblement plu aux Crétois; en effet, ce sont trois courts hommages ${ }^{167}$ qui ont été placés à Herculanum aux frais de la province ${ }^{168}$. L'un de ces hommages ${ }^{169}$ provient sans doute du chalcidicum de la basilique. Mais, fait encore plus intéressant, deux cités crétoises ont ressenti le besoin d'honorer, elles aussi, le patron provincial - peut-être d'autres cités ont-elles agi de même -; or, comme le fait remarquer M.A. Baldwin ${ }^{170}$, il s'agit de Cnossos $^{171}$ et de Gortyne ${ }^{172}$, qui ont toutes deux bénéficié de la politique octavo-augustéenne, la toute nouvelle colonie de Cnossos étant fondée par Octavien ${ }^{173}$ et Gortyne devenant la capitale provinciale ${ }^{174}$. Pour cet auteur, ces deux cités auraient été les clientes ${ }^{175}$ de M. Nonius Balbus, ce qui n'est pas impossible. Ces cinq témoignages d'une relation patronale très appréciée par les clients ${ }^{176}$ ont coûté fort cher: en plus de l'inscription elle-même, il a fallu payer le transport de la pierre, ou plus sûrement l'acheter localement - l'une des bases ${ }^{177}$

(163) RE, XVII, 1, 1936, s. u. Nonius, col. 900-901, nº 52; Perl G., Klio, 52, 1970, p. 332, n 6; Marshall A. J., ANRW, I, 1, 1972, p. 901, note 54; HARrison G., Cretan Studies, 1, 1988, p. 141. Il a été préteur avant 53 av. J.-C.; cf. STELLA Maranca F., Memorie della R. Accademia nazionale dei Lincei, Classe di scienze morali storiche e filologiche, ser. VI, II, 4, 1927, p. 332-333.

(164) Paribeni R., dans DE, II, 2, 1910, s. u. Creta, col. 1260-1261.

(165) Cf. annexe 1, doc. a; b.

(166) Cf. annexe 1, doc. c.

(167) Ce ne sont évidemment pas les Crétois qui ont voté à M. Nonius Balbus des honneurs funéraires importants, contrairement à ce qu'affirme A. PAutasso (ASAA, 72/73, 1994/95, p. 77), qui confond les Crétois dédicants avec l'ordo d'Herculanum (MaIUri A., Atti della reale Accademia d'Italia, Rendiconti della classe di Scienze morali e storiche, ser. 7, III, 1943, p. 253-278 (AE, 1947, 53) = Schumacher L., Chiron, 6, 1976, p. 165-184 (AE, 1976, 144) Herculanum, Regio I. Hors les murs, dans le cortile des thermes dits de «Porta Marina». Ara marmorea, retrouvée conjointement avec la tête d'une statue de M. Nonius Balbus, ce qui indique la localisation de son tombeau: [Qu]od M(arcus) Ofillius Celer, (duum)uir iter(um) $u($ erba) f(ecit): pertinere at municipi / dignitatem meritis $M($ arci) Noni Balbi respondere, $d(e)$ e (a) $r(e) i(t a) c(e n s u e r u n t)$. I $[\mathrm{Cu}] \mathrm{m}$ M(arcus) Nonius Balbus quo hac uixerit parentis animum cum plurima liberalitat(e) / singulis uniuersisque praistiterit, placere decurionibus statuam equestrem ei poni quam / celeberrimo loco ex pecunia publica inscribique: M(arco) Nonio M(arci) f(ilio) Balbo, pr(aetori), proco(n)s(uli), patrono, uniuersus / ordo populi Herculaniessis ob merita eius; item eo loco, quo cineres eius conlecti sunt, aram / marmoream fieri et constitui inscribique publice: $M$ (arco) Nonio $M($ arci) $f(i l i o)$ Balbo; exque eo loco parentalibu(s) / pompam duci ludisque gymnicis qui soliti erant fieri diem adici unum in honorem eius et cum in theatro / ludi fiunt sellam eius poni. C(ensuere).).

(168) Maiuri A., Atti della reale accademia d'Italia. Rendiconti della classe di scienze morali e storiche, ser. 7, III, 1943, p. 270.

(169) Cf. annexe 1, doc. a.

(170) BALdwin M. A., Fasti, 1983, p. 88. On peut signaler qu'elle n'évoque à aucun moment le patronat provincial.

(171) Cf. annexe 1, doc. d.

(172) Cf. annexe 1, doc. e.

(173) Cf. ci-dessus, p. 262. L’hommage a sans doute été rendu après le retour de M. Nonius Balbus en Italie, et la réussite de sa mission de patron provincial. Il est donc normal que la cité de Cnossos indique son statut de colonie, même si celui-ci ne lui a été attribué qu'au début de l'année 27 av. J.-C..

(174) Harrison G., Cretan Studies, 1, 1988, p. 144-148s; Levick B. M., CAH², X, 1996, p. 648; Haensch R., Capita, 1996 , p. $202 ; 350$, note 158 .

(175) BALdwin-Bowsky M.A., CJ, 82, 1987, p. 220.

(176) Baldwin-Bowsky M.A., CJ, 82, 1987, p. 219 sur l'importance des hommages: "No other proconsul of this province is known to have been so honored, with provincial dedications erected in his Italian hometown ». Cf. MAIURI A., Atti della reale accademia d'Italia. Rendiconti della classe di scienze morali e stroriche, ser. 7, III, 1943, p. 263.

(177) Cf. annexe 1, doc. a. 
était surmontée d'une statue équestre en bronze -, envoyer des délégués à Herculanum, chargés de négocier avec l'ordo local des localisations, faire accepter le texte par le dédicataire, trouver un lapicide, inaugurer les documents, ce qui signifie un séjour assez long dans la baie de Naples. Mais il semble que le coût était considéré comme nécessaire, car c'est une quête publique -aere collatoqui finance l'hommage des habitants de Gortyne, preuve que l'ensemble de la population a voulu participer à ce témoignage de réciprocité.

Ce dossier met bien en lumière l'importance du contexte local dans la nomination d'un patron de province: un certain nombre de caractéristiques sont mises ici en évidence, comme le choix d'un personnage connaissant la province, ou / et ayant des relations privilégiées avec le Prince. M. Nonius Balbus, proconsul puis patron de la Crète après la victoire d'Actium et avant le découpage provincial de 27 av. J.-C., a permis à cette province de s'intégrer dans l'empire romain, réunifié par OctavienAuguste, en témoignant de sa fidélité au Prince. Son intercession auprès d'Auguste explique sans doute l'attribution de la province, pacifiée et fidélisée, au Sénat et au peuple romain.

\section{Sabine LEFEBVRE \\ Université de Bourgogne - UMR 6298 ARTeHIS}

\section{Annexe 1. Les hommages rendus à M. Nonius Balbus par les Crétois}

a) CIL, X, $1430=2405$ = Guadagno G., Cronache Ercolanesi, 11, 1981, p. 143, nº 91 Herculanum, Regio I. Base de statue équestre en bronze découverte sans doute dans le chalcidicum de la basilique. Epistylium. [M(arco) Nonio M(arci)f(ilio)] Balbo, proco $(n) s(u l i) ; /[$ commune] Cretensium, patrono.

b) CIL, X, 1431 Herculanum, Regio I. M(arco) Nonio M(arci) f(ilio) / Bal[bo], pr(aetori), proco(n)s(uli); / [commune Creten] sium.

c) CIL, X, 1432 Herculanum, Regio I. M(arco) Noni[o M(arci)f(ilio)] / Bal[b]o, pr(aetori), p[roco(n)s(uli)]; / commune C[retensium].

d) CIL, X, 1433 Herculanum, Regio I. M(arco) Nonio M(arci)f(iio) / Balbo, pr(aetori), proco(n)s(uli); / colonia Iulia C[nossus?].

e) CIL, X, 1434 Herculanum, Regio I. M(arco) Nonio M(arci) f(ilio) / Balbo, pr(aetori), proco(n)s(uli); / Gortyniei ae[re coll(ato)].

Annexe 2. Les monnaies du pseudo P. Licinius

La présence en Crète de P. Aemilius Lepidus en 43 av. J.-C. est attestée par des monnaies qui avaient été attribuées à un P. Licinius en raison de leur très mauvais état de conservation ${ }^{178}$; la présence de ce personnage posait un certain nombre de difficultés, en particulier en ce qui concerne la nature des fonctions remplies - pro $q$ (uaestor) pr(o praetore)? - et la date de sa présence - vers 55 av. J.-C., si on l'identifie avec P. Licinius P.f. Crassus Iunianus? ou l'époque antonienne? Plusieurs types étaient connus:

Doc 1. BMC Cyr., p. cciii, $\mathrm{n}^{\circ} 2$ bis a; p. 113, n² 2, pl. XXXIX, 5 (photographie) = Chapman A., Num. Chron., 8, 1968, p. 15, pl. V, 3 :

sur le droit, tête de Libya, diadémée et buste drapé, à droite, la légende est en très mauvais état: on peut deviner $\Lambda \mathrm{I}-\mathrm{B} \Psi \mathrm{H}$ de chaque côté du buste et P. L[i]CI[niu? ... ] autour; un cercle de points borde la pièce;

sur le revers, buste de Créta-Artémis à droite avec devant la lettre B, et la légende KPHTA; avec autour, [p] LI[cinius p]RO Q PR[...].

Doc 2. BMC Cyr., p. cciii, $\mathrm{n}^{\circ} 2$ bis a; p. 113, $\mathrm{n}^{\circ} 2$ bis, pl. XXXIX, 6 (photographie):

sur le droit, tête de Libya, diadémée et buste drapé, à droite, la légende est en très mauvais état: on peut deviner $\Lambda \mathrm{I}-\mathrm{B} \Psi \mathrm{H}$ de chaque côté du buste et [lici]NIVS [...]PRO Q autour; un cercle de points borde la pièce;

sur le revers, buste de Créta-Artémis à droite, avec la légende $[\chi \rho]$ HTA B; avec autour, $[\ldots]$ LIC $[\ldots]$. 
Mais un autre exemplaire de cette monnaie, conservé à Berlin, a permis d'améliorer la lecture de la légende du revers, désormais attribuée à P. Lepidus, mentionné par Appien, et daté de 43 av. J.-C.; P. Licinius n'est en effet pas attesté en Crète, mais aurait seulement été pro quaestor de Cyrénaïque ${ }^{179}$ :

Doc 3: Grant M., Imperium, 1946, pl. II, 15 : la légende serait P LEPID [p f pro q]).

Mais la présentation de M. Grant est incorrecte; en effet, la légende P. LEPID doit être attribuée, non au revers, mais au droit où figure la Libye. C'est donc en Libye que P. Lepidus aurait été en poste ${ }^{180}$. Depuis, E. A. Sydenham a mentionné ces monnaies, maintenant la localisation de Lepidus en Crète ${ }^{181}$.

Trois autres monnaies ${ }^{182}$ ont permis de modifier une seconde fois la lecture: le personnage mentionné serait P. Lepidius pour les deux faces, et donc les deux provinces; mais aucun P. Lepidius P. f. en fonction n'est connu dans les deux provinces conjointement. La série a sans doute été frappée en Crète, et peut être attribuée à la période triumvirale, vers 40 av. J.-C. ${ }^{183}$. La monnaie ayant permis la meilleure lecture confirme cette modification (Doc 4: RPC, I, p. 219, $\mathrm{n}^{\circ}$ 907, pl. 51, $\mathrm{n}^{\circ}$ 907).

\section{Annexe 3. Les monnaies de L. Pinarius Scarpus}

Doc 1. Babelon E., Monnaies, II, 1885, p. 305; 306, n 9 et $10=\mathrm{I}$, p. 198, ${ }^{\circ} 98$ et 99 (dessin) = BMC Rep., II, 1910, p. 584, $\mathrm{n}^{\circ} 2$ et $3=$ Sydenham E. A., Coinage, 1952, p. 200-201, $\mathrm{n}^{\circ} 1280$ et $1280 \mathrm{a}=R R C$, p. $542-543, \mathrm{n}^{\circ} 546, \mathrm{n}^{\circ} 2$ et 3 : sur le droit, la tête de Jupiter Ammon à droite et la légende M(arco) ANTO(nio) CO(n)S(ul) III IMP(erator) IIII; sur le revers, une Victoire marchant à droite, tenant une couronne et une palme, avec la légende ANTONIO AVG(uri), SCARPVS

Doc 2. Babelon E., Monnaies, II, 1885, p. 306, nº $11=\mathrm{I}$, p. 198, $\mathrm{n}^{\circ} 100($ dessin$)=$ BMC Rep., II, p. 583-584, $\mathrm{n}^{\circ} 1=$ Sydenham E.A., Coinage, 1952, p. 200-201, nº $1279=R R C$, p. 542-543, nº 546, $\mathrm{n}^{\circ} 1$ et pl. LXIV, $\mathrm{n}^{\circ} 17$ : sur le droit, la tête de Jupiter Ammon à droite et la légende M(arco) ANTO(nio) Co(n)s(ul) III IMP(erator) IIII; sur le revers, l'aigle légionnaire entre deux enseignes de cohortes, avec la légende SCARPVS IMP(erator), LEG(io) VIII.

Doc 3. Cohen H., Médailles, I, 1880, p. 82, n $125=$ BMC Rep., II, p. 584, n 4 = RIC, I, p. $44=$ BMC Emp., I, p. 112, n 690, pl. $17, \mathrm{n}^{\circ} 18$ = Sydenham E. A., Coinage, 1952, p. 201, n $1281=C B N$, I, p. 142, nº 897 et 898, pl. XXXIV en 29 av. J.-C. $=R I C, \mathrm{I}^{2}, 1984$, p. $84, \mathrm{n}^{\circ} 535$, entre 31 et 29 av. J.-C. $=R R C$, p. 542-543, $\mathrm{n}^{\circ} 546, \mathrm{n}^{\circ} 4$ et pl. LXIV, $\mathrm{n}^{\circ} 18$ : sur le droit la tête de Jupiter Ammon à droite et la légende AVGVR / PONTIF(ex); sur le revers une Victoire sur un globe à droite, tenant une couronne et une palme, avec la légende IMP(erator) CAESAR / DIVI F(ilius).

Doc 4. Cohen H., Médailles, I, 1880, p. 136, n $500=$ BAbelon E., Monnaies, II, 1885 , p. 306, $\mathrm{n}^{\circ} 12($ dessin $)=$ p. $60, \mathrm{n}^{\circ} 142=$ BMC Rep., II, p. 585, n 5 = RIC, I, p. 44, pl. III, n $60=$ BMC Emp., I, p. 111, nº 689, pl. 17, 16 = SydenhAM E. A., Coinage, 1952 , p. $201, \mathrm{n}^{\circ} 1282=R R C$, p. $542-543, \mathrm{n}^{\circ} 546, \mathrm{n}^{\circ} 5$ et 6 et pl. LXIV, $\mathrm{n}^{\circ} 19=C B N, \mathrm{I}, \mathrm{p} .141, \mathrm{n}^{\circ} 894-896$, pl. XXXIV $=R I C, \mathrm{I}^{2}, 1984$, p. $84, \mathrm{n}^{\circ} 533$ et 534 :

sur le droit, main ouverte avec la légende IMP(eratori) CAESARI / SCARPVS IMP(erator);

sur le revers, une Victoire tenant une palme et une couronne, debout sur un globe et tournée à droite avec la légende DIVI F(ilio) / AVG(uri) PONT(ifici). Il est daté de 31 av. J.-C. ${ }^{184}$.

Doc 5. Cohen H., Médailles, $I$, 1880, p. 136, n 498 = Babelon E., Monnaies, II, 1885, p. 307, $\mathrm{n}^{\circ} 13$ (dessin) = p. 60, n $143=$ BMC Rep., II, p. 585-586, nº 7 et $8=$ RIC, I, p. 44 = BMC Emp., I, p. 111, n 686 = Sydenham E. A., Coinage, 1952 , p. $201, \mathrm{n}^{\circ} 1283=R R C$, p. $542-543, \mathrm{n}^{\circ} 7=C B N, \mathrm{I}, \mathrm{p} .141, \mathrm{n}^{\circ} 891$, pl. XXXIV $=R I C, \mathrm{I}^{2}, 1984, \mathrm{p} .84, \mathrm{n}^{\circ} 531$ : sur le droit, main ouverte avec la légende SCARPVS IMP(erator); sur le revers, une Victoire tenant une palme et une couronne, debout sur un globe et tournée à droite avec la légende CAESARI DIVI F(ilio).

(179) Grant M., Imperium, 1946, p. 35-36

(180) Chapman A., Num. Chron., 8, 1968, p. 15-16, note 3, qui conserve néanmoins dans sa liste le nom de Licinius.

(181) Sydenham E. A., Coinage, 1952, p. 203; 214: têtes de Libya ou de Creta respectivement et la légende, P LEPID P F PRO Q LIBYH ou P LEPID P F PRO Q PR [...] KPHTA.

(182) Price M., INJ, 6/7, 1982/83, p. 119, note 5; RPC, I, p. 219, n 907 : au droit, buste de Libya, avec $\Lambda$ IBYH et la légende P LEPIDIVS PRO Q; au revers, buste d'Artémis à droite avec un arc et KPHTA ou KP-HTA et la légende P LEPIDIVS P F PRO Q, avec des variantes.

(183) RPC, I, p. 217-218; 219.

(184) Cet aureus, conservé au musée de Narbonne, a fait l'objet d'une publication récente: Crawford M. et RichARD J.-C., RAN, 34, 2001, p. 166-169, avec de belles photographies. 
Doc 6. Cohen H., Médailles, $I, 1880$, p. 136, n $499=$ BAbelon E., Monnaies, II, 1885, p. 307, $\mathrm{n}^{\circ} 14$ (dessin) = p. 60, $\mathrm{n}^{\circ} 144=$ BMC Rep., II, p. 586, n 6 = BMC Emp., $I$, p. 111, nº 687, pl. 17, 17 = Sydenham E. A., Coinage, 1952, p. 201, $\mathrm{n}^{\circ} 1284=$ $R R C$, p. 542-543, $\mathrm{n}^{\circ} 8=C B N$, I, p. $141, \mathrm{n}^{\circ} 892-893$, pl. XXXIV $=R I C, \mathrm{I}^{2}, 1984, \mathrm{p} .84, \mathrm{n}^{\circ} 532$ :

sur le droit, main ouverte avec la légende SCARPVS IMP(erator);

sur le revers, une Victoire tenant une palme et une couronne, debout sur un globe et tournée à droite avec la légende CAESAR[i] DIVI F(ilio).

\section{Annexe 4. Monnaies mentionnant des gouverneurs de Crète ou de Cyrénaïque.}

Doc 1. Scato.

Falbe C. T., Lindberg J. C. et Müller L., Numismatique, 1860, p. 166-167, n 430-433; BMC Cyr., p. ccviii; ccxxiiiccxxiv; p. 119-120, n 36-43, pl. XLIII, n 7-10; Grant M., Imperium, 1946, p. 137-139; Buttrey T. V., dans Studies in numismatic Method presented to Philip Grierson, 1983, p. 27-28; 32 ; RPC, I, p. 225 (entre 20 et 12 av. J.-C.); p. 228, $\mathrm{n}^{\circ} 942$ et $943, \mathrm{pl} .53, \mathrm{n}^{\circ} 942$ et 943 (aes):

au droit, tête nue d'Auguste à droite avec en légende CAESAR TR POT;

au revers, une sella castrensis avec un coussin sur le siège, et la légende SCATO PR.

Doc 2. A. Pupius Rufus.

BMC Cyr., p. 118, n²9, pl. XLIII, $1=$ RPC, I, p. 222, nº 920, pl. 52, n 920 :

au droit, tête d'Ammon à droite, avec deux cornes sur son front, derrière la tête, KAI TAMIAX $\Lambda$;

au revers, sella castrensis avec des coussins sur le siège, de chaque côté, fasces; au-dessus et en-dessous, П] OYПI[OC ANTICTPAT L.

Doc 3. Capito.

Falbe C. T., Lindberg J. C. et Müller L., Numismatique, 1860, p. 168-169, nº 438; BMC Cyr., p. ccvii-ccviii, $\mathrm{n}^{\circ} 48 \mathrm{~b}$; ccxxv; pl. XLIV, $\mathrm{n}^{\circ} 5=, I, 1992$, p. 227, $\mathrm{n}^{\circ} 938$, pl. 53, $\mathrm{n}^{\circ} 938$ :

au droit, couronne de laurier avec IMP AVG / TR POT;

au revers sella castrensis avec un coussin sur le siège et CAPITO au dessus, en-dessous, Q(uaestor):

\section{Annexe 5. Les monnaies de Kydas.}

Le crétarque ${ }^{185}$ d'époque antonienne est connu par plusieurs types monétaires ${ }^{186}$, qui sont ci-dessous présentés. Le document 1 est attribué à Kydas, placé parmi les magistrats de Cnossos, et non à Kydas, crétarque.

Doc 1. BMC Cret., 1886, p. 24-25, n 57, pl. VI, 10 = Chapman A., Num. Chron., 8, 1968, p. 17, pl. VI, $7=$ RPC, I, p. 224, $\mathrm{n}^{\circ} 934$, pl. 53, n 934 :

au droit, tête de Zeus lauré à droite;

au revers, aigle avec les ailes ouvertes debout à droite; la légende $\mathrm{K} \Psi \Delta \mathrm{A} \Sigma$.

On peut en particulier mentionner un cistophore de Gortyne longtemps daté entre 67/66 et 31 av. J.-C., et plus précisément de 44 av. J.-C. ${ }^{187}$, mais qu'il faut aujourd'hui placer après la période de $37 / 36$ av. J.-C.:

Doc 2. Imhoof-Blumer F., Monnaies, 1883, p. 210-211, $\mathrm{n}^{\circ} 1$ = BMC Cret., 1886, p. xix-xx = Svoronos J., Numismatique, 1890, p. 334, nº 1 = BMC Cyr., p. ccix, note 2= HeAD B. V., HN², 1963, p. 479= Chapman A., Num. Chron., 8, 1968, p. $17 ; 20-21$ et pl. V, $9=R P C$, I, p. 224, n 926 , pl. 52, $\mathrm{n}^{\circ} 926$ :

au droit, une couronne de lierre avec au centre, une ciste entr'ouverte d'où s'échappe un serpent à gauche;

au revers, entre deux serpents dressés et affrontés, Zeus Krétagénès nu debout à droite, lançant le foudre de la main droite et tenant à gauche un aigle s'envolant à droite; au dessus, $K \Psi \triangle \mathrm{A} \Sigma$, au dessous, KPHTAPXA $\Sigma$ et dans le champ, $\mathrm{KPH}-\mathrm{TAI} / \mathrm{E}-\Omega$ / N.

\section{Annexe 6. Les magistrats de la colonie de Cnossos dans les monnaies augustéennes}

$\mathrm{Au}$ droit de ces monnaies figure Agrippa et au revers Octavien ; les légendes mentionnent les trois couples de magistrats de la nouvelle colonie de Cnossos.

(185) Nous avons tenu pour acquis que le Kydas crétarque, le Kydas mentionné par Cicéron, et le Kydas figurant sur les monnaies étaient un seul et même personnage. Pour les discussions sur le sujet, cf. Paluchowski A., Elites, 2004, p. 206-209.

(186) Wroth W., BMC Cret., 1886, p. 24-25, n 53-60= Chapman A., Num. Chron., VIII, 1968, p. 17; 21; pl. VI, 7; VII, 3 et 6 = Burnett A., Amandry M., Pau Ripollès P., RPC, I, p. 224, nº 934, pl. 53, n 934; Paluchowski A., Elites, 2004, p. 204-205.

(187) Imноof-Blumer F., Monnaies, 1883, p. 210-211, nº 1, en particulier p. 211, note 7. 
Doc 1. Monnaies de T. Fufius et M. Aemilius: BMC Cret., 1886, p. 26, n 72 , pl.VI, 12; Svoronos J., Numismatique, 1890, p. 89-90, nº 183, pl. VIII, 11 et 12:

au droit, tête d'Agrippa, anciennement attribuée à Marc Antoine, à droite, tête nue, derrière sa tête, un petit labyrinthe carré rectiligne avec autour de la tête, la légende: [C I]N C [EX D D ?];

au revers, tête d'Octavien à droite, tête nue avec la légende T. FVFIO devant, M. AIMI[LIO] derrière et dessous [IIVIR].

Doc 2. Monnaies de C. Petronius et M. Antonius:

Svoronos J., Numismatique, 1890, p. 90, n 188 et 189, pl. VIII, 13 et 14 :

au droit, tête d'Auguste à droite, tête nue; la légende: $\mathrm{C}$ I N / CN;

au revers, labyrinthe carré avec autour, la légende [C] PETRON[IO] au dessus, M ANTONIO au dessous, IIVIR à droite, EX DD à gauche.

Doc 3. Monnaies de Aeschinus Caes. L iter et Plotius Plebeius:

au droit, tête nue d'Auguste avec au dessus, AVGVSTO et C N I CNOS au dessous; EX / DD à travers le champ;

au revers, Rome vêtue d'une tunique courte debout à gauche tenant dans la main une petite Niké qui la couronne et dans la main gauche une haste; en légende circulaire AESHINO CAES L ITER PLOTIO PLEB IIVIR et dans le champ, RO/MA.

Doc 4. Monnaie d'Auguste:

Svoronos N., Numismatique, 1890, p. 90, $\mathrm{n}^{\circ} 185$, pl. VIII, 7 = RPC, I, p. 237, n 985, pl. 55, $\mathrm{n}^{\circ} 985$ :

au droit, charrue à droite avec en dessous D D AVG;

au revers, labyrinthe carré rectiligne.

\section{Bibliographie}

Adamo Muscettola S., «Nuove letture borboniche: i Nonii Balbi ed il Foro di Ercolano», Prospettiva, 28, 1982, p. 2-16.

Álvarez y Saenz de Buruaga J., «La fundación de Mérida,» dans Blanco Freijeiro A. (éd.), Augusta Emerita. Actas del Symposio Internacional conmemorativo del bimilenario de Mérida, noviembre 1975, Madrid, 1976, p. 19-32.

-, «Acerca del nombre de la colonia Augusta Emerita», Museos, 1, 1982, p. 5-7.

Babelon E., Description historique et chronologique des monnaies de la république romaine vulgairement appelées monnaies consulaires, Paris, 1885.

Baldwin M.A., Fasti Cretae et Cyrenarum. Imperial Magistrates of Crete and Cyrenaica during the JulioClaudian Period, Ph. D. University of Michigan, 1983.

Baldwin-Bowsky M.A., "Roman Arbitration in Central Crete. An Augustean Proconsul and a Neronian Procurator», CJ, 82, 1987, p. 218-229.

Bastianini G., «Lista dei prefetti d'Egitto dal 30 av. J.-C. al 299 ap. J.-C.», ZPE, 17, 3, 1975, p. 263-328.

—, «Lista dei prefetti d'Egitto dal 30 av. J.-C. al 299 ap. J.-C. Aggiunte e correzioni», ZPE, 38, 1980, p. 75-89.

—, «Il prefetto d'Egitto (30 a. C.-297 d. C.). Addenda (1973-1985)», ANRW, II, 10, 1, Berlin, 1988, p. 503-517.

Bertrand J.-M., «Rome et la Méditerranée orientale au premier siècle av. J.-C.», dans Nicolet C. (éd.), Rome et la conquête du monde méditerranéen, 2, Genèse d'un empire, Paris, 1978, p. 789-845.

Bowersook G., Augustus and the Greek World, Oxford, 1965.

Brulé P., La piraterie crétoise hellénistique, Annales littéraires de l'Université de Besançon 223, Paris, 1978.

Bureth P., «Le préfet d'Egypte (30 av. J.-C.-297 ap. J.-C.) : état présent de la documentation en 1973 », ANRW, II, 10, 1, Berlin, 1988, p. 472-502.

Buttrey T. V., "Thea Neotera on Coins of Antony and Cleopatra », ANSMN, Museum Notes, VI, 1954, p. 95-109.

-, «Roman Coinage of the Cyrenaica, first century B.C. to first century A. D.» dans Brooke C. N. L., Stewart B.H.I.H., Pollard J.G. et Volk T.R. (éd.), Studies in Numismatic Method presented to Philip Grierson, Cambridge, 1983, p. 23-46. 
-, "Crete and Cyrene», dans Burnett A.M. et Crawford M.H. (éd.), The Coinage of the Roman World in the Late Republic, Proceedings of a Colloquium held at the British Museum in September 1985, BAR International series 326, Oxford, 1987, p. 165-174.

Cabanes P., «Le monde grec européen et la Cyrénaïque», dans Lepelley C. (éd.), Rome et l'intrégration de l'Empire 44 av. J.-C./260 ap. J.-C., 2. Approches régionales du Haut-Empire romain, Paris, 1998, p. 299-331.

Caillemer E., «Cretensium respublica,» dans Daremberg C. et Saglio E., Dictionnaire, I-2, Paris, 1887, p. $1563-1568$.

Camodeca G., «Ascesa al senato e rapporti con i territori d'origine italia: regio I (Campania, esclusa la zona di Capua e Cales), II (Apulia et Calabria), III (Lucania et Brutti)», EOS, II, Tituli, 5, Rome, 1982, p. 101-163.

Canto A. M., "Colonia Iulia Augusta Emerita. Consideraciones entorno a su fundación y territorio », Gerión, 7, 1989, p. 149-205.

-, «Las tres fundaciones de Augusta Emerita», dans Trillmich W. et Zanker P. (éd.), Stadtbild und Ideologie. Die Monumentalisierung hispanicher Städte zwischen Republik und Kaiserzeit. Kolloquium im Madrid, 19-23 oktober 1987, Munich, 1990, p. 289-297.

Chamoux F., Marc Antoine, Paris, 1986.

Chapman A., «Some first Century B. C. Bronze Coins of Knossos», Num. Chron., 7e s., 8, 1968, p. 13-26.

Charlesworth M.P., "The Avening of Caesar», CAH, X, The Augustan Empire 44 B.C.-A.D. 70, Cambridge, 1963, p. 1-30.

Cohen H., Description historique des monnaies frappées sous l'empire romain, communément appelées médailles impériales, I, Paris-Londres, 1880.

Constans L. A. et Bayet J. (trad.), Cicéron. Correspondance, tome IV, CUF, Paris, 1962.

Crawford M., Roman Republican Coins Hoards, Londres, 1969.

—, «CR de K. Kraft, Zür münzprägung des Augustus, Wiesbaden, 1969», JRS, 64, 1974, p. 246-247.

Crawford M. et Richard J.-C., «L'aureus de Lucius Pinarius Scrapus (31 av. J.-C.) découvert à Narbonne (Aude)», RAN, 34, 2001, p. 166-169.

Daremberg C. et Saglio E., Dictionnaire des Antiquités grecques et romaines d'après les textes et les monuments, Paris, 1877-1919.

D’Hugues G., Une province romaine sous la république. Étude sur le proconsulat de Cicéron, Paris, 1876.

De Laet S., De Samenstelling van den römeinschen senaat gedurende de eerste eeuw van het principaat, Anvers, 1941.

Deininger J., Die Provinziallandtage der römischen Kaiserzeit von Augustus bis zum Ende des dritten Jahrhunderts n. Chr., Munich, 1965.

Dobiás J., "La donation d'Antoine à Cléopâtre en l'an 34 av. J.-C.», dans Mél. Bidez, I, Annuaire de l'institut de philologie et d'histoire orientales, 2, Bruxelles, 1934, p. 287-314.

Ducrey P., «Trois nouvelles inscriptions crétoises», BCH, 93, 1969, p. 841-852.

Eck W., «Über die prätorischen Prokonsulate in der Kaiserzeit. Eine quellenkritische überlegung», Zephyrus, 23/24, 1972/73, p. 233-260.

Falbe C. T., Lindberg J. C. et Müller L., Numismatique de l'ancienne Afrique, I, Les monnaies de la Cyrénaïque, Paris-Leipzig-Copenhague, 1860.

Frei P., «Zur Gründung und zur Rechtsstellung der römischen Kolonie Aventicum», Pro Aventico, 20, 1969, p. 5-22.

Freyburger M.-L. et Roddaz J.-M. (trad.), Dion Cassius, Histoire romaine, Livres 48-49, CUF, Paris, 1994.

Gardthausen V., Augustus und seine Zeit, Leipzig, 1891/1904.

Gasperini L., «Le iscrizioni del Cesareo e della basilica di Cirene», QAL, 6, 1971, p. 3-22.

Grant M., From Imperium to Auctoritas: a Historical Study of Aes Coinage in the Roman Empire 49 B. C.-A. D. 14, Cambridge, 1946. 
Grimal P., Cicéron, Paris, 1986.

Guarducci M., «Un personaggio della gens Rubellia onorato a Gortina,» Bulletino comunale di Roma, 56, 1928, p. 275-283.

—, «Osservazioni intorno al trattato fra Hierapytna e Priansos,», Epigraphica, 2, 1940, p. 149-166.

Guiraud P., Les assemblées provinciales dans l'Empire romain, Paris, 1887.

Gurval R. A., Actium and Augustus. The Politics and Emotions of Civil War, Ann Arbor, 1995.

Haensch R., Capita provinciarum. Statthaltersitze und provinzialverwaltung in der römischen Kaiserzeit, Forschungen 7, Mayence, 1997.

Harrison G., «Background to the first Century of Roman Rule in Crete», Cretan Studies, 1, 1988, p. 125-155.

Head B. V., $H N^{2}=$ Historia numorum. A manual of Greek numismatics ${ }^{2}$, Londres, 1963.

Hölzl M., Fasti praetorii ab a. u. DCLXXXVII usque ad a. u. DCCX, Diss. Leipzig, 1876.

Hurlet F., Les collègues du prince sous Auguste et Tibère, De la légalité républicaine à la légitimité dynastique, CEFR 227, Rome, 1997.

Imhoof-Blumer F., Monnaies grecques, Amsterdam, 1883.

Jal P. (trad.), Tite Live, Histoire romaine, XXXII. Livres XLIII-XLIV, CUF, Paris, 1976.

Jones A.H.M., The Greek City from Alexandrer to Justinian, Oxford, 1967 (1940).

Kraft K., Zür Münzprägung des Augustus, Wiesbaden, 1969 (=Castricius H. et Kienast D. (éd.), Kleine Schriften, II: Gesammelte Aufsätze zur antiken Geldgeschichte und Numismatik, I, Darmstadt, 1978, p. 291-337).

Laronde A., Cyrène et la Libye hellénistique. $\Lambda$ YBIKAI ILTOPIAI de l'époque républicaine au principat d'Auguste, Paris, 1987.

—, «La Cyrénaïque romaine des origines à la fin des Sévères (96 av. J.-C.-235 ap. J.-C.)», ANRW, II, 10, 1, Berlin, 1988, p. 1006-1064.

Larsen J.A. O., "Cyrene and the Panhellenion », Classical Philology, 47, 1952, p. 7-16.

Lassère F. (trad.), Strabon. Géographie, tome VII (livre X), CUF, Paris, 1971.

Lefebvre S., Patronus prouinciae, à paraître.

Levick B. M., «Greece (includind Crete and Cyprus) and Asia Minor from 43 B.C. to A. D. 69 », $C A H^{2}$, X, The Augustean Empire, 43 B.C.-A.D. 69, Cambridge, 1996, p. 641-675.

Luzzatto G. I., Roma e le province. I. Organissazione, economia, società, Storia di Roma, XVII-1, Bologne, 1985. Magie D., Roman Rule in Asia Minor to the End of the Third Century after Christ, Princeton, 1950 (RRAM).

Maiuri A., «Un decreto onorario di M. Nonio Balbo scoperto recentemente ad Ercolano,» Atti della reale accademia d'Italia. Rendiconti della classe di scienze morali e storiche, ser. 7, III, 1943, p. 253-282.

Malitz J., «O puer qui omnia nomini debes». Zur Biographie Octavians bis zum Antritt seines Erbes», Gymnasium, 111, 2004, p. 381-409.

Marshall A.J., "The Lex Pompeia de provinciis (52 B.C.) and the Cicero's Imperium in 51-50 B.C.: Constitutionnal Aspects», ANRW, I, 1, Berlin, 1972, p. 887-921.

Müller L., Numismatique de l'ancienne Afrique. Supplément, Copenhague, 1874.

Münzer F., «Aus dem Verwandtenkreise Caesars und Oktavians», Hermes, 71, 1936, p. 222-230.

Muttelsee M., Zur Verfassungsgeschichte Kretas im Zeitalter des Hellenismus, Glückstadt-Hambourg, 1925.

Nicols J., «Patrons of Greek Cities in the Early Principate», ZPE, 80, 1990, p. 81-100.

Oost S. I., «Cyrene, 96-74 BC», Classical Philology, 58, 1963, p. 11-25.

Paluchowski A., Les élites urbaines en Crète sous le Haut-Empire, Tours, Tomes 1 et 2, 2004.

Paribeni R, «Creta», dans De Ruggiero E., Dizionario epigrafico di Antichità romane, Spolète, 1910, II, 2, col. 1257-1275.

Pauly A. et Wissowa G., Realencyclopädie der classischen Altertumswissenschaft, Stuttgart, 1890.

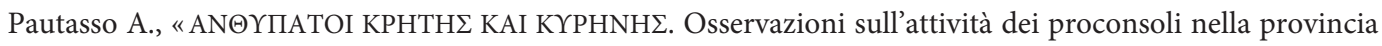
nei primi secoli dell'impero", ASAA, 72/73, 1994/95, p. 75-108.

Pelling C., «The Triumviral Period», $C A H^{2}$, X, The Augustean Empire, 43 B.C.-A.D. 69, Cambridge, 1996, p. 1-69. 
Perl G., «Die römischen Provinzbeamten in Cyrenae und Creta zur Zeit der Republik», Klio, 52, 1970, p. 319-354.

—, «Die römischen Provinzbeamten in Cyrenae und Creta zur Zeit der Republik. Nachträge zum Beitrag», Klio, 53, 1971, p. 369-379.

Perrot G., "Cretarchia», dans Daremberg C. et Saglio E., Dictionnaire des Antiquités grecques et romaines..., I-2, Paris, 1887, p. 1562-1563.

Pflaum H.-G., «Le règlement successoral d'Hadrien», dans Bonner Historia Augusta Colloquium 1963, Antiquitas, R. 4, Bd. 2, Bonn 1964, p. 95-122.

Price M., «Crete, Cyrene, and Dio LII, 30, 9», INJ, 6/7, 1982/83, p. 118-127.

Raven E. J.P., «The Hierapytnian Hoard of Greek and Roman Coins», Num. Chron., 18, 1938, p. 133-158.

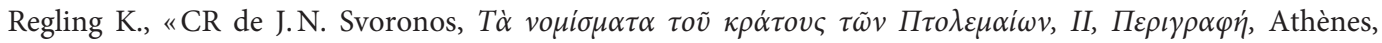
1904 », ZfN, 25, 1906, p. 344-399.

Rémy B., «L'activité des proconsuls et des représentants du prince dans la province de Crète-Cyrénaïque au Haut-Empire d'après les inscriptions», dans Laronde A. et Maffre J.-J. (éd.), Cités, ports et campagnes de la Cyrénaïque gréco-romaine, 21 novembre 1992, Karthago, 24, 1999, p. 165-186.

Reynolds J. et Llyod J.A., "Cyrene,», $C_{A H}^{2}$, X, The Augustean Empire, 43 B.C.-A.D. 69, Cambridge, 1996, p. 619-640.

Rigsby K. J., «Cnossos et Capua», TAP, 106, 1976, p. 313-330.

Roddaz J.-M., Marcus Agrippa, CEFR 253, Rome, 1984.

Romanelli P., «Egypt, Crete and Cyrenaica, III. Crete», CAH, XI, The Imperial Peace A.D. 70-192, Cambridge, 1936, p. 659-675.

—, La Cirenaica romana (96 a. C./642 d.C.), Verbania, 1943.

Rouanet-Liesenfelt, A.-M., "Le crétarque Kydas", dans Aux origines de l'hellénisme, la Crète et la Grèce, Hom. à H. van Effenterre, Paris, 1984, p. 343-352.

—, «Remarques sur l'assemblée provinciale crétoise et son grand-prêtre à l'époque du Haut-Empire», Ktèma, 19, 1994, p. 7-26.

Salmon E. T., Roman Colonization under the Republic, Londres, 1949.

Sanders I. F., Roman Crete. An Archeological Survey and Gazetteer of Late Hellenistic, Roman and Early Byzantine Crete, Warminster, 1982.

Sartori F., «Il commune Siciliae nel tardo impero », Klio, 63, 1981, 2, p. 401-409.

Sartre M., L'Orient romain. Provinces et sociétés provinciales en Méditerranée orientale d'Auguste aux Sévères (31 av. J.-C. $/ 235$ ap. J.-C.), Paris, 1991.

Schumacher L., «Das Ehrendekret für M. Nonius Balbus aus Herculaneum (AE, 1947, 53)», Chiron, 6, 1976, p. 165-184.

Shackleton Bailey D. R., Cicero's letters to Atticus, III, 51-50 B. C., Cambridge, 1968.

Sherwin-White A.N., "Lucullus, Pompey and the east», CAH', IX, The Last Age of the Roman Republic, 146-43 B.C., Cambridge, 1994, p. 229-273.

Spyridakis S., Ptolemaic Itanos and Hellenistic Crete, Berkeley, 1970.

Stella Maranca F., «Fasti praetorii, I, dal 366 al 44 av. Cristo», Memorie della R. Accademia nazionale dei Lincei, Classe di scienze morali storiche e filologiche, ser. 6, II, 4, 1927, p. 279-376.

Sternkopf W., «Die Verteilung der römischen Provinzen vor dem mutinensischen Kriege», Hermes, 47, 1912, p. 321-401.

Svoronos J.N., Numismatique de la Crète ancienne accompagnée de l'Histoire, la géographie et la mythologie de l'île, Macon, 1890.

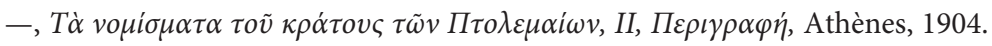

Sydenham E. A., The Coinage of the Roman Republic, Londres, 1952.

Syme R., The Augustean Aristocracy, Oxford, 1986. 
Szramkiewicz R., Les gouverneurs de province à l'époque augustéenne, Paris, I, 1975; II, 1976.

Talbert R. J. A., The Senate of Imperial Rome, Princeton, 1984.

Tarn W.W. et Charlesworth M.P., «The War of the East against the West», CAH, X, The Augustan Empire 44 B.C.-A.D. 70, Cambridge, 1963, p. 66-111.

Thomasson B. E., Laterculi Praesidum, III, Göteborg, 1990.

—, Laterculi Praesidum, I, Göteborg, 2009.

Thompson D. J., «Egypt 146-31 B.C.», CAH², IX, The Last Age of the Roman Republic, 146-43 B.C., Cambridge, 1994, p. 310-326.

Tzamtzis I.E, «Antoine, Cléopâtre et la Crète. À propos de Dion Cassius, 49, 32, 4-5», dans Traiana G. (éd.), Studi sull'età di Marco Antonio, Rudiae, Ricerche sul mondo classico, 18, 2006, p. 353-375.

Van der Mijnsbrugge M., The Cretan Koinon, Amsterdam-New-York, 1931.

Van Effenterre H., La Crète et le monde grec de Platon à Polybe, BEFAR 163, Paris, 1948.

Vittinghoff P., Römische Kolonisation und Bürgerrechtspolitik unter Caesar und Augustus, Abhand. Akad. Des Wiss., Wiesbaden, 1951.

Weidemann U., «Eine Bemerkung zu C. Rubellius Blandus cos. suff. 18, und dessen Vater», Acta Classica, 7 , 1964, p. 64- 69.

Willets R. F., Aristocratic Society in Ancient Crete, Londres, 1955.

—, «The Cretan Koinon: Epigraphy and Tradition», Kadmos, 14, 1975, p. 143-148.

Zucca R., «Un nuovo procurator prouinciae Cyrenarum », dans Catani E. et Marengo S. M. (éd.), La Cirenaica in etàantica, Attidel convegno internazionale distudi, Macerata, 18-20 mars 1995, Macerata, 1998, p. 623-637. 